This item was submitted to Loughborough's Research Repository by the author.

Items in Figshare are protected by copyright, with all rights reserved, unless otherwise indicated.

\title{
Power extraction in regular and random waves from an OWC in hybrid wind- wave energy systems
}

\section{PLEASE CITE THE PUBLISHED VERSION}

https://doi.org/10.1016/j.oceaneng.2019.106519

\section{PUBLISHER}

Elsevier

\section{VERSION}

AM (Accepted Manuscript)

\section{PUBLISHER STATEMENT}

This paper was accepted for publication in the journal Ocean Engineering and the definitive published version is available at https://doi.org/10.1016/j.oceaneng.2019.106519.

\section{LICENCE}

CC BY-NC-ND 4.0

\section{REPOSITORY RECORD}

Michele, Simone, Emiliano Renzi, Carlos Perez-Collazo, Deborah Greaves, and Gregorio Iglesias. 2019. "Power Extraction in Regular and Random Waves from an OWC in Hybrid Wind-wave Energy Systems". figshare. https://hdl.handle.net/2134/9917933.v1. 


\title{
Power extraction in regular and random waves from an OWC in hybrid wind-wave energy systems
}

\author{
S.Michele ${ }^{\mathrm{a}, *}$, E.Renzi $^{\mathrm{a}}$, C.Perez-Collazo ${ }^{\mathrm{b}}$, D.Greaves ${ }^{\mathrm{c}}$, G.Iglesias ${ }^{\mathrm{d}, \mathrm{c}}$ \\ ${ }^{a}$ Department of Mathematical Sciences, Loughborough University, Leics LE11 3TU, UK \\ ${ }^{b}$ School of Mining and Energy Engineering, University of Vigo, Vigo, Pontevedra 36310, \\ Spain \\ ${ }^{c}$ School of Engineering, University of Plymouth, Drake Circus, Plymouth PL4 8AA, UK \\ ${ }^{d}$ Environmental Research Institute 83 School of Engineering, University College Cork, \\ Western Rd., Cork, Ireland
}

\begin{abstract}
A mathematical model is developed to analyse the hydrodynamics of a novel oscillating water column (OWC) in a hybrid wind-wave energy system. The OWC has a coaxial cylindrical structure in which the internal cylinder represents the mono-pile of an offshore wind turbine while the external cylinder has a skirt whose scope is to guide the wave energy flux inside the chamber. This layout is not casual, but consistent with the current approach to harnessing wave energy through hybrid systems. The device shape is rather complex and the boundary value problem is solved by applying the matchingmethod of eigenfunctions. Within the framework of a linearised theory, we model the turbine damping effects by assuming the airflow to be proportional to the air chamber pressure. Consequently, the velocity potential can be decomposed into radiation and diffraction problems. We study the effects of both skirt and internal radius dimensions on the power extraction efficiency for monochromatic and random waves. We show that the skirt has strong effects on the global behaviour, while the internal cylinder affects the values of the sloshing eigenfrequencies. Finally, we validate the analytical model with laboratory data and show a good agreement between analytical and
\end{abstract}

\footnotetext{
${ }^{*}$ Corresponding author

Email addresses: s.michele@lboro.ac.uk (S.Michele), e.renzi@lboro.ac.uk (E.Renzi), carlos.perez.collazo@uvigo.es (C.Perez-Collazo), deborah.greaves@plymouth.ac.uk (D.Greaves), gregorio.iglesias@ucc.ie (G.Iglesias)
} 
experimental results.

Keywords: Wave energy, Wave-structure interactions, Oscillating water column

\section{Introduction}

The oscillating water column (OWC) is one of the most studied devices to extract energy from water waves. For an extensive review concerning related theories and experiments we refer to the works of McCormick (1981), Falnes (2002), Babarit et al. (2012) and Babarit (2018). Substantially, the OWC is a partially-immersed structure open at its bottom that confines air above the internal fluid free-surface. Incident waves induce oscillations inside the chamber, thus the airflow is forced to pass through a turbine usually located at the top of the OWC. The turbine is coupled to a generator to produce electricity. In this paper we perform a novel analysis of a coaxial cylindrical OWC in a hybrid wind-wave energy system.

Analytical theories concerning immersed floating structures open at one end have been applied in several contexts. One of the main contributions is due to Garrett (1970), who examined cylindrical bottomless harbours. Other authors such as Mavrakos (1985) analysed the effects of the wall thickness of a floating cylindrical body on the diffracted wave field. Extension of hydrodynamical theories to OWC devices includes the analysis of both bidimensional (Evans, 1982; Sarmento and Falcão, 1985) and three-dimensional configurations. For example, Evans and Porter (1997) were the first to solve the case of a partially immersed cylindrical OWC in open sea by applying the Galerkin method to integral equations.

Several recent studies consider the OWC to be installed in fixed structures for coastal protection. This is mainly due to economical reasons and difficulties in developing wave energy absorbers on their own. Concerning analytical models for OWCs combined with external structures, Martin-Rivas and Mei (2009a) and Martin-Rivas and Mei (2009b) solved the linearised problem of an OWC at the tip of a breakwater and the case of an OWC installed on a straight coast. More recently, Lovas et al. (2010) extended the theory of Martin-Rivas and Mei (2009b) to examine the hydrodynamic wave field when the OWC is installed in correspondence of convex or concave corners, Deng et al. (2013) and Deng et al. (2014) took into account the presence of a coaxial supporting structure to examine possible benefits on the energy 
conversion efficiency, while Zhou et al. (2018) solved the case of a concentric axisymmetric OWC including an internal mono-pile. Wave-structure interaction in hybrid wave farms with OWC devices was investigated by Zheng et al. (2018), while recently, breakwater-integrated OWCs were studied by means of a semi-analytical model by Zheng et al. (2019).

Despite the large number of theories developed so far, the wave energy sector is suffering from slow technological progress combined with difficulties in attracting funds (Magagna and Uihlein, 2015). This is mainly due to the large levelised cost of energy (LCOE) (Astariz et al., 2015, 2016). One way to attract funds and confidence in industry is to combine both wind and wave energy technologies. This is a recent research effort that aims to develop a more sustainable and affordable device to extract clean energy against fossil fuels. We refer to the work of Perez-Collazo et al. (2015) for an extensive review of alternatives that combine wave and offshore wind energy technologies.

Motivated by this recent technology concept, Perez-Collazo et al. (2018a) and Perez-Collazo et al. (2018b) tested a 1:50 novel hybrid wind-wave system that integrates a skirt, a cylindrical OWC and an offshore wind turbine on a jacket frame structure. The Authors investigated experimentally the hydrodynamic response of the device to monochromatic and random waves for different orifice diameters simulating different air turbines. Promising results were obtained; however further analytical work is needed to understand the influence of the device parameters on the global hydrodynamic behaviour of the system.

For all these reasons, in this paper we develop a mathematical model for the OWC designed by Perez-Collazo et al. (2018b) by adding a concentric cylinder that represents a wind turbine installed on a mono-pile. This system appears similar to the theoretical models already solved by the Authors previously mentioned. However, the skirt connected to the OWC removes any axial symmetry and the boundary value problem increases in complexity.

Here we apply an eigenfunction expansion method and solve the corresponding velocity potentials in terms of Bessel functions and modified Bessel functions of the first and second kind, respectively (Linton and McIver, 2001; Mei et al., 2005). First, we analyse the case of monochromatic waves and derive several integral relations based on the Green's theorem which can be useful to check the numerical computations of the radiation and diffraction velocity potentials. Then we show that both the skirt and the internal cylinder play an important role in the power extraction and the sloshing dynamics 
inside the air chamber.

Next, we extend the theory to the case of random waves described by the JONSWAP spectrum (Goda, 2000) and characterise the power extraction efficiency by applying the superposition principle to the different incident wave frequency components. We find that the resonant peaks related to the Helmholtz and sloshing modes decrease in intensity with respect to the monochromatic case, and that the the random waves have a broadening effect on the capture factor curve. Interestingly, similar results were also found in the context of oscillating wave surge converters (Michele et al., 2016a,b; Sarkar et al., 2014).

Laboratory experimental models usually simulate the damping effects of a turbine by means of an orifice of a certain diameter connecting the air chamber with the atmosphere (Perez-Collazo et al., 2018b). In this case, the air turbine is of the impulse type; therefore, a quadratic relation exists between the airflow through the orifice and the pressure head between the air chamber and the atmosphere, thus the boundary condition on the free surface becomes nonlinear (Pereiras et al., 2015; López et al., 2016, 2014). We then non-dimensionalise the corresponding equation by using adequate scales (Michele et al., 2018, 2019a; Michele and Renzi, 2019; Sammarco et al., 1997a,b) and apply a perturbation technique to the velocity potential. We show that if the ratio between the orifice and OWC diameter is not very small, the air pressure inside the chamber and the corresponding airflow through the orifice are governed by the diffraction potential at the leading order. Finally, we compare our analytical model with the 1:50 scale model in Perez-Collazo et al. (2018b) and show good matching of the theoretical results with those evaluated experimentally.

\section{Governing Equations}

With reference to figure 1, consider an OWC device embedded in a hybrid wave-wind energy extraction system. Let us define a Cartesian reference system with the $x$ and $y$-axes coincident with the undisturbed free-surface level and the $z$-axis pointing vertically upward. The concentric cylindrical structure of the OWC has inner radius equal to $R_{i}$, while the external radius corresponds to $R_{e}$. The internal cylinder spans the entire water depth and is fixed with the horizontal bottom at $z=-h$. The external cylinder does not have constant draught in water but includes a skirt of height $h_{s}$ and corresponding arc-length $R_{e}\left(\theta_{2}-\theta_{1}\right)$ with $\theta_{2}>\theta_{1}$. The remaining part of 

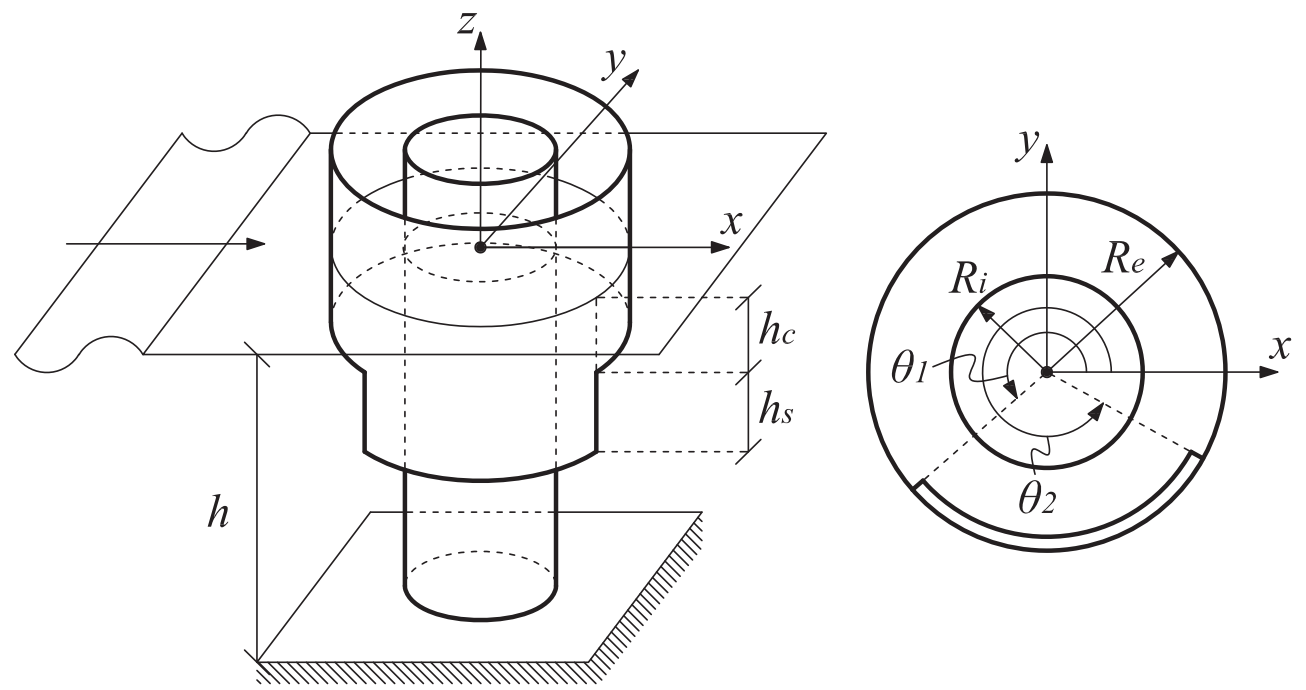

Figure 1: Three-dimensional representation and horizontal cross-section of the hybrid wind-wave energy converter.

the external structure has draught equal to $h_{c}$. The chamber is open at its base, while at the top an energy conversion system transforms the airflow through it in electricity (Falnes, 2002). Let us define the solid wetted surfaces of the OWC

$$
\begin{aligned}
S_{R_{i}} & =\left\{r=R_{i}, \theta \in[0,2 \pi), z \in[-h, 0]\right\}, \\
S_{R_{e}} & =\left\{r=R_{e}, \theta \in[0,2 \pi), z \in\left[-h_{c}, 0\right]\right\} \\
& \cup\left\{r=R_{e}, \theta \in\left[\theta_{1}, \theta_{2}\right], z \in\left[-h_{c}-h_{s},-h_{c}\right]\right\},
\end{aligned}
$$

and the fluid surface $S_{f}$ representing the gap under the OWC,

$$
S_{f}=\left\{r=R_{e}, \theta \in[0,2 \pi), z \in[-h, 0]\right\} \backslash S_{R_{e}},
$$

where $r=\sqrt{x^{2}+y^{2}}$ represents the radial coordinate and $\theta$ is the angular coordinate positive anticlockwise. Moreover, let us define the following surfaces

$$
\begin{gathered}
S_{i}=\left\{r \in\left[R_{i}, R_{e}\right], \theta \in[0,2 \pi), z=0\right\}, \\
S_{e}=\left\{r \in\left[R_{e}, \infty\right), \theta \in[0,2 \pi), z=0\right\},
\end{gathered}
$$

where $S_{i}$ denotes the free surface inside the chamber, while $S_{e}$ is the external free surface in contact with air at constant atmospheric pressure. The fluid 
is inviscid and incompressible, while the fluid flow can be assumed to be irrotational. Then the governing equation for the velocity potential $\Phi(x, y, z, t)$ satisfies the Laplace equation in the fluid domain $\Omega(x, y, z)$. On the free surfaces $S_{e}$ and $S_{i}$ we have the linearised kinematic condition

$$
\zeta_{t}=\Phi_{z}, \quad z=0
$$

and the linearised mixed boundary conditions (Mei et al., 2005)

$$
\begin{aligned}
& \Phi_{t t}+g \Phi_{z}=0, \quad \text { on } S_{e}, \\
& \Phi_{t t}+g \Phi_{z}=-\frac{P_{a_{t}}}{\rho}, \quad \text { on } S_{i},
\end{aligned}
$$

where $\zeta$ represents the free-surface elevation, $g$ is the acceleration due to gravity, $P_{a}$ denotes the oscillating pressure of the air inside the chamber depending on time $t$ and $\rho$ is the water density. We further require tangential fluid velocity at the bottom and on the solid surfaces, hence

$$
\Phi_{n}=0, \quad \text { on solid boundaries, }
$$

where $n$ denotes the normal derivative to the relevant surface. The problem is forced by monochromatic incident waves of frequency $\omega$, hence let us assume harmonic motion

$$
\left\{\Phi, \zeta, P_{a}\right\}=\operatorname{Re}\left\{\left(\phi, \eta, p_{a}\right) e^{-\mathrm{i} \omega t}\right\}
$$

with i being the imaginary unit. We shall now write the Laplace equation and the boundary conditions (6)-(9) in terms of the spatial variables $\left(\phi, \eta, p_{a}\right)$ only. Thus we get

$$
\begin{aligned}
\nabla^{2} \phi & =0, & & \text { in } \Omega, \\
-\mathrm{i} \omega \eta & =\phi_{z}, & & z=0, \\
-\omega^{2} \phi+g \phi_{z} & =0, & & \text { on } S_{e}, \\
-\omega^{2} \phi+g \phi_{z} & =\mathrm{i} \omega \frac{p_{a}}{\rho}, & & \text { on } S_{i}, \\
\phi_{n} & =0, & & \text { on solid boundaries . }
\end{aligned}
$$

Finally we require that the velocity potential $\phi$ be outgoing for $r \rightarrow \infty$. 
141

Following the method of Evans and Porter (1997) we decompose the velocity potential in two parts, i.e.

$$
\phi=\phi^{D}+\phi^{R},
$$

where $\phi^{D}$ is the diffraction potential satisfying the boundary conditions (12)(15) with $p_{a}=0$ and $\phi^{R}$ is the radiation potential that satisfies the same conditions with the unknown forcing pressure $p_{a} \neq 0$. Let us decompose the fluid domain $\Omega$ by defining $\Omega_{i}$ and $\Omega_{e}$, respectively, as the internal and external fluid subdomains:

$$
\Omega_{i}=\left\{r \in\left(R_{i}, R_{e}\right), \theta \in[0,2 \pi), z \in(-h, 0)\right\},
$$

$$
\Omega_{e}=\left\{r \in\left(R_{e}, \infty\right), \theta \in[0,2 \pi), z \in(-h, 0)\right\},
$$

and let $\phi_{i}^{D}\left(\phi_{i}^{R}\right)$ be the diffraction (radiation) potential in $(r, \theta, z) \in \Omega_{i}$ and $\phi_{e}^{D}\left(\phi_{e}^{R}\right)$ the diffraction (radiation) potentials in $(r, \theta, z) \in \Omega_{e}$.

The boundary value problem for the external velocity potentials $\phi_{e}^{D, R}$ is

$$
\begin{aligned}
\nabla^{2} \phi_{e}^{D, R} & =0, & & \text { in } \Omega_{e}, \\
-\omega^{2} \phi_{e}^{D, R}+g \phi_{e_{z}}^{D, R} & =0, & & \text { on } S_{e}, \\
\phi_{e_{r}}^{D, R} & =0, & & \text { on } S_{R_{e}} \\
\phi_{e_{z}}^{D, R} & =0, & & z=-h \\
\phi_{e}^{D, R} & =\phi_{i}^{D, R}, & & \text { on } S_{f}, \\
\phi_{e_{r}}^{D, R} & =\phi_{i_{r}}^{D, R}, & & r=R_{e}
\end{aligned}
$$

where the conditions (23)-(24) represent respectively continuity of the potential (pressure) and of the velocity field between the external velocity potentials $\phi_{e}^{D, R}$ and the internal velocity potentials $\phi_{i}^{D, R}$. The boundary value problem for the internal velocity potentials $\phi_{i}^{D, R}$ is governed by

$$
\begin{aligned}
\nabla^{2} \phi_{i}^{D, R} & =0, & & \text { in } \Omega_{i}, \\
-\omega^{2} \phi_{i}^{D}+g \phi_{i_{z}}^{D} & =0, & & \text { on } S_{i}, \\
-\omega^{2} \phi_{i}^{R}+g \phi_{i_{z}}^{R} & =\mathrm{i} \omega \frac{p_{a}}{\rho}, & & \text { on } S_{i}, \\
\phi_{i_{r}}^{D, R} & =0, & & \text { on } S_{R_{i}}, \\
\phi_{i_{r}}^{D, R} & =0, & & \text { on } S_{R_{e}}, \\
\phi_{i_{z}}^{D, R} & =0, & & z=-h,
\end{aligned}
$$


and the coupling matching conditions (23)-(24).

In the following sections we solve the diffraction and radiation potential in $\Omega_{e}$ and $\Omega_{i}$ by integrating the matching conditions on the common boundaries.

\subsection{Diffraction potential solution}

Let us assume for simplicity incident waves with direction parallel to the $x$-axis and amplitude $A$. The generalized angles $\theta_{1}$ and $\theta_{2}$ can be properly modified in order to investigate the effects of oblique incident waves. For the sake of example, a skirt described by the angles $\bar{\theta}_{1}=\theta_{1}+\alpha$ and $\bar{\theta}_{2}=\theta_{2}+\alpha$ simulates the effects of incoming waves with angle of incidence $\pi-\alpha$ on the same OWC.

Use of cylindrical coordinates yields the following general solution for the diffraction potential in $(r, \theta, z) \in \Omega_{e}$

$$
\begin{aligned}
& \phi_{e}^{D}=-\frac{\mathrm{i} A g}{\omega} \sum_{n=0}^{\infty}\left\{\frac { \operatorname { c o s h } k _ { 0 } ( h + z ) } { \operatorname { c o s h } k _ { 0 } h } \left\{\mathcal{B}_{n 0}^{D} \frac{\sin n \theta H_{n}^{(1)}\left(k_{0} r\right)}{\left.H_{n_{r}}^{(1)}\left(k_{0} r\right)\right|_{r=R_{e}}}\right.\right. \\
& \left.+\cos n \theta\left[\epsilon_{n} \mathrm{i}^{n} J_{n}\left(k_{0} r\right)+\mathcal{A}_{n 0}^{D} \frac{H_{n}^{(1)}\left(k_{0} r\right)}{\left.H_{n_{r}}^{(1)}\left(k_{0} r\right)\right|_{r=R_{e}}}\right]\right\}+ \\
& \left.\sum_{l=1}^{\infty} \frac{\left(\mathcal{A}_{n l}^{D} \cos n \theta+\mathcal{B}_{n l}^{D} \sin n \theta\right) K_{n}\left(\bar{k}_{l} r\right) \cosh k_{l}(h+z)}{\left.K_{n_{r}}\left(\bar{k}_{l} r\right)\right|_{r=R_{e}} \cosh k_{l} h}\right\},
\end{aligned}
$$

while the diffraction potential in $(r, \theta, z) \in \Omega_{i}$ can be written as

$$
\begin{aligned}
\phi_{i}^{D} & =-\frac{\mathrm{i} A g}{\omega} \\
& \times \sum_{n=0}^{\infty}\left\{\frac { \operatorname { c o s h } k _ { 0 } ( h + z ) } { \operatorname { c o s h } k _ { 0 } h } \left\{\cos n \theta\left[\mathcal{C}_{n 0}^{D} \frac{J_{n}\left(k_{0} r\right)}{\left.J_{n_{r}}\left(k_{0} r\right)\right|_{r=R_{i}}}+\mathcal{D}_{n 0}^{D} \frac{Y_{n}\left(k_{0} r\right)}{\left.Y_{n_{r}}\left(k_{0} r\right)\right|_{r=R_{i}}}\right]\right.\right. \\
& \left.+\sin n \theta\left[\mathcal{E}_{n 0}^{D} \frac{J_{n}\left(k_{0} r\right)}{\left.J_{n_{r}}\left(k_{0} r\right)\right|_{r=R_{i}}}+\mathcal{F}_{n 0}^{D} \frac{Y_{n}\left(k_{0} r\right)}{\left.Y_{n_{r}}\left(k_{0} r\right)\right|_{r=R_{i}}}\right]\right\} \\
& +\sum_{l=1}^{\infty} \frac{\cosh k_{l}(h+z)}{\cosh k_{l} h}\left\{\cos n \theta\left[\mathcal{C}_{n l}^{D} \frac{I_{n}\left(\bar{k}_{l} r\right)}{\left.I_{n_{r}}\left(\bar{k}_{l} r\right)\right|_{r=R_{i}}}+\mathcal{D}_{n l}^{D} \frac{K_{n}\left(\bar{k}_{l} r\right)}{\left.K_{n_{r}}\left(\bar{k}_{l} r\right)\right|_{r=R_{i}}}\right]\right. \\
& \left.\left.+\sin n \theta\left[\mathcal{E}_{n l}^{D} \frac{I_{n}\left(\bar{k}_{l} r\right)}{\left.I_{n_{r}}\left(\bar{k}_{l} r\right)\right|_{r=R_{i}}}+\mathcal{F}_{n l}^{D} \frac{K_{n}\left(\bar{k}_{l} r\right)}{\left.K_{n_{r}}\left(\bar{k}_{l} r\right)\right|_{r=R_{i}}}\right]\right\}\right\} .
\end{aligned}
$$


In the latter expressions, $A$ denotes the amplitude of the incident waves, $\epsilon_{n}$ is the Jacobi symbol defined as

$$
\epsilon_{0}=1, \quad \epsilon_{n}=2 \quad n=1, \ldots, \infty,
$$

the terms $k_{l}$ 's are the roots of the dispersion relation (Mei et al., 2005)

$$
\left.\begin{array}{l}
\omega^{2}=g k_{0} \tanh k_{0} h, \\
\omega^{2}=-g \bar{k}_{l} \tan \bar{k}_{l} h, \quad k_{l}=\mathrm{i} \bar{k}_{l}, \quad l=1, \ldots, \infty
\end{array}\right\}
$$

$J_{n}$ and $Y_{n}$ are the Bessel functions of order $n, H_{n}^{(1)}$ is the Hankel function of the first kind and order $n, I_{n}$ and $K_{n}$ are the modified Bessel functions of order $n$ and finally $\mathcal{A}_{n l}^{D}, \mathcal{B}_{n l}^{D}, \mathcal{C}_{n l}^{D}, \mathcal{D}_{n l}^{D}, \mathcal{E}_{n l}^{D}, \mathcal{F}_{n l}^{D}$ are complex constants yet unknown. The no-flux condition (28) yields

$$
\mathcal{C}_{n l}^{D}=-\mathcal{D}_{n l}^{D}, \quad \mathcal{E}_{n l}^{D}=-\mathcal{F}_{n l}^{D}
$$

thus expression (32) reads now

$$
\begin{aligned}
\phi_{i}^{D} & =-\frac{\mathrm{i} A g}{\omega} \sum_{n=0}^{\infty}\left\{\frac{\cosh k_{0}(h+z)}{\cosh k_{0} h}\left(\cos n \theta \mathcal{D}_{n 0}^{D}+\sin n \theta \mathcal{F}_{n 0}^{D}\right) \mathcal{T}_{n}\right. \\
& \left.+\sum_{l=1}^{\infty} \frac{\cosh k_{l}(h+z)}{\cosh k_{l} h}\left(\cos n \theta \mathcal{D}_{n l}^{D}+\sin n \theta \mathcal{F}_{n l}^{D}\right) \mathcal{U}_{n l}\right\},
\end{aligned}
$$

where

$$
\mathcal{T}_{n}=\frac{Y_{n}\left(k_{0} r\right)}{\left.Y_{n_{r}}\left(k_{0} r\right)\right|_{r=R_{i}}}-\frac{J_{n}^{(1)}\left(k_{0} r\right)}{\left.J_{n_{r}}\left(k_{0} r\right)\right|_{r=R_{i}}}, \mathcal{U}_{n l}=\frac{K_{n}\left(\bar{k}_{l} r\right)}{\left.K_{n_{r}}\left(\bar{k}_{l} r\right)\right|_{r=R_{i}}}-\frac{I_{n}\left(\bar{k}_{l} r\right)}{\left.I_{n_{r}}\left(\bar{k}_{l} r\right)\right|_{r=R_{i}}} .
$$

171 Substituting expressions (36) and (31) in the matching condition (24) and 172 integrating over $S_{f} \cup S_{R_{e}}$, gives

$$
\left.\epsilon_{n} \mathrm{i}^{n} J_{n_{r}}\left(k_{0} r\right)\right|_{r=R_{e}}+\mathcal{A}_{n 0}^{D}=\left.\mathcal{D}_{n 0}^{D} \mathcal{T}_{n_{r}}\right|_{r=R_{e}}, \quad \mathcal{B}_{n 0}^{D}=\left.\mathcal{F}_{n 0}^{D} \mathcal{T}_{n_{r}}\right|_{r=R_{e}},
$$

$$
\mathcal{A}_{n l}^{D}=\left.\mathcal{D}_{n l}^{D} \mathcal{U}_{n l_{r}}\right|_{r=R_{e}}, \quad \mathcal{B}_{n l}^{D}=\left.\mathcal{F}_{n l}^{D} \mathcal{U}_{n l_{r}}\right|_{r=R_{e}} .
$$


174 175

$$
\begin{aligned}
\phi_{e}^{D} & =-\frac{\mathrm{i} A g}{\omega} \sum_{n=0}^{\infty}\left\{\frac { \operatorname { c o s h } k _ { 0 } ( h + z ) } { \operatorname { c o s h } k _ { 0 } h } \left\{\operatorname { c o s } n \theta \left[\epsilon_{n} \mathrm{i}^{n} J_{n}\left(k_{0} r\right)+\frac{H_{n}^{(1)}\left(k_{0} r\right)}{\left.H_{n_{r}}^{(1)}\left(k_{0} r\right)\right|_{r=R_{e}}}\right.\right.\right. \\
& \left.\left.\times\left(\left.\mathcal{D}_{n 0}^{D} \mathcal{T}_{n_{r}}\right|_{r=R_{e}}-\left.\epsilon_{n} \mathrm{i}^{n} J_{n_{r}}\left(k_{0} r\right)\right|_{r=R_{e}}\right)\right]+\left.\mathcal{F}_{n 0}^{D} \mathcal{T}_{n_{r}}\right|_{r=R_{e}} \frac{\sin n \theta H_{n}^{(1)}\left(k_{0} r\right)}{\left.H_{n_{r}}^{(1)}\left(k_{0} r\right)\right|_{r=R_{e}}}\right\} \\
& \left.+\left.\sum_{l=1}^{\infty} \frac{\cosh k_{l}(h+z)}{\cosh k_{l} h} \frac{K_{n}\left(\bar{k}_{l} r\right)}{\left.K_{n_{r}}\left(\bar{k}_{l} r\right)\right|_{r=R_{e}}} \mathcal{U}_{n l_{r}}\right|_{r=R_{e}}\left(\mathcal{D}_{n l}^{D} \cos n \theta+\mathcal{F}_{n l}^{D} \sin n \theta\right)\right\}
\end{aligned}
$$

178

179

181

For the sake of brevity, we introduce the following integrals for the vertical eigenfunctions

$$
\mathrm{I}_{s l}^{(1)}=\int_{-h}^{-h c-h s} \frac{\cosh k_{l}(h+z) \cosh k_{s}(h+z)}{\cosh k_{l} h \cosh k_{s} h} \mathrm{~d} z,
$$

$$
\mathrm{I}_{s l}^{(2)}=\int_{-h c-h s}^{-h c} \frac{\cosh k_{l}(h+z) \cosh k_{s}(h+z)}{\cosh k_{l} h \cosh k_{s} h} \mathrm{~d} z,
$$

$$
\mathrm{I}_{s l}^{(3)}=\int_{-h c}^{0} \frac{\cosh k_{l}(h+z) \cosh k_{s}(h+z)}{\cosh k_{l} h \cosh k_{s} h} \mathrm{~d} z,
$$

and the following integrals involving the angular eigenfunctions

$$
\mathrm{cc}_{p n}=\int_{\theta_{2}}^{\theta_{1}} \cos n \theta \cos p \theta \mathrm{d} \theta, \quad \mathrm{ss}_{p n}=\int_{\theta_{2}}^{\theta_{1}} \sin n \theta \sin p \theta \mathrm{d} \theta,
$$

$$
\mathrm{sc}_{p n}=\int_{\theta_{2}}^{\theta_{1}} \sin n \theta \cos p \theta \mathrm{d} \theta, \quad \mathrm{cs}_{p n}=\int_{\theta_{2}}^{\theta_{1}} \cos n \theta \sin p \theta \mathrm{d} \theta,
$$

2 whose values can be found straightforwardly. 
Multiplying the condition $(23)$ by $\cosh k_{s}(h+z) \cos p \theta / \cosh k_{s} h$ and integrating over the fluid surface $S_{f}$, yields

$$
\begin{aligned}
& \frac{4 \mathrm{I}_{s 0}^{(1)} \epsilon_{n} \mathrm{i}^{n+1} \delta_{p n}}{\left.\epsilon_{p} R_{e} H_{n_{r}}^{(1)}\left(k_{0} r\right)\right|_{r=R_{e}}}+\sum_{m=0}^{\infty} \frac{2 \mathrm{cc}_{p m} \mathrm{I}_{s 0}^{(2)} \epsilon_{m} \mathrm{i}^{m+1}}{\left.R_{e} \pi H_{m_{r}}^{(1)}\left(k_{0} r\right)\right|_{r=R_{e}}}=\frac{2 \mathcal{D}_{n 0}^{D} \mathrm{I}_{s 0}^{(1)} \delta_{p n} \pi \tau_{n}}{\epsilon_{p}} \\
& +\sum_{m=0}^{\infty} \mathrm{I}_{s 0}^{(2)} \tau_{m}\left(\mathcal{D}_{m 0}^{D} \mathrm{cc}_{m n}+\mathcal{F}_{m 0}^{D} \mathrm{Sc}_{p m}\right)+\sum_{l=1}^{\infty} \frac{2 \mathcal{D}_{n l}^{D} \mathrm{I}_{s l}^{(1)} \delta_{p n} \pi \gamma_{n l}}{\epsilon_{p}} \\
& +\sum_{m=0}^{\infty} \sum_{l=1}^{\infty} \mathrm{I}_{s 0}^{(2)} \gamma_{m l}\left(\mathcal{D}_{m l}^{D} \mathrm{cc}_{p m}+\mathcal{F}_{m l}^{D} \mathrm{sc}_{p m}\right), \quad s=0,1, \ldots, p=0,1, \ldots,
\end{aligned}
$$

where $\delta_{p n}$ is the Kronecker delta, while $\tau_{m}$ and $\gamma_{m l}$ have the following expressions

$$
\tau_{m}=\left.\mathcal{T}_{m}\right|_{r=R_{e}}-\left.\frac{\left.H_{m}^{(1)}\left(k_{0} r\right)\right|_{r=R_{e}}}{\left.H_{m_{r}}^{(1)}\left(k_{0} r\right)\right|_{r=R_{e}}} \mathcal{T}_{m_{r}}\right|_{r=R_{e}}
$$

$$
\gamma_{m l}=\left.\mathcal{U}_{m l}\right|_{r=R_{e}}-\left.\frac{\left.K_{m}\left(\bar{k}_{l} r\right)\right|_{r=R_{e}}}{\left.K_{m_{r}}\left(\bar{k}_{l} r\right)\right|_{r=R_{e}}} \mathcal{U}_{m l_{r}}\right|_{r=R_{e}}
$$

Multiplying again the condition (23) by $\cosh k_{s}(h+z) \sin p \theta / \cosh k_{s} h$ and integrating over the fluid surface $S_{f}$ gives now

$$
\begin{aligned}
& \pi \mathcal{F}_{n 0}^{D} \mathrm{I}_{s 0}^{(1)} \delta_{p n} \tau_{n}+\sum_{m=0}^{\infty} \mathrm{I}_{s 0}^{(2)} \tau_{m}\left(\mathcal{D}_{m 0}^{D} \mathrm{cs}_{p m}+\mathcal{F}_{m 0}^{D} \mathrm{SS}_{p n}\right) \\
& +\sum_{l=1}^{\infty} \pi \mathcal{F}_{n l}^{D} \mathrm{I}_{s l}^{(1)} \delta_{p n} \gamma_{n l}+\sum_{m=0}^{\infty} \sum_{l=1}^{\infty} \mathrm{I}_{s l}^{(2)} \gamma_{m l}\left(\mathcal{D}_{m l}^{D} \mathrm{cs}_{p m}+\mathcal{F}_{m l}^{D} \mathrm{ss}_{p m}\right)=0 .
\end{aligned}
$$

An additional condition for the internal diffraction potential can be obtained by multiplying $\phi_{i, r}^{D}=0$ respectively by $\cosh k_{s}(h+z) \cos p \theta / \cosh k_{s} h$ and $\cosh k_{s}(h+z) \sin p \theta / \cosh k_{s} h$. Integrating over the relevant domain $S_{R_{e}}$ gives us

$$
\begin{aligned}
& \sum_{l=1}^{\infty} \frac{\left.2 \pi \mathcal{D}_{n l}^{D} \mathrm{I}_{s l}^{(3)} \delta_{p n} \mathcal{U}_{n l_{r}}\right|_{r=R_{e}}}{\epsilon_{p}}+\left.\sum_{m=0}^{\infty} \sum_{l=1}^{\infty} \mathrm{I}_{s l}^{(2)} \mathcal{U}_{m l_{r}}\right|_{r=R_{e}}\left(\mathcal{D}_{m l}^{D} \overline{\mathrm{cc}}_{p m}+\mathcal{F}_{m l}^{D} \overline{\mathrm{sc}}_{p m}\right) \\
& +\frac{\left.2 \pi \delta_{p n} \mathrm{I}_{s 0}^{(3)} \mathcal{D}_{n 0}^{D} \mathcal{T}_{n_{r}}\right|_{r=R_{e}}}{\epsilon_{p}}+\left.\sum_{m=0}^{\infty} \mathrm{I}_{s 0}^{(2)} \mathcal{T}_{m_{r}}\right|_{r=R_{e}}\left(\mathcal{D}_{m 0}^{D} \overline{\mathrm{cc}}_{p m}+\mathcal{F}_{m 0}^{D} \overline{\mathrm{sc}}_{p m}\right)=0,
\end{aligned}
$$




$$
\begin{aligned}
& \left.\pi \delta_{p n} \mathrm{I}_{s 0}^{(3)} \mathcal{F}_{n 0}^{D} \mathcal{T}_{n_{r}}\right|_{r=R_{e}}+\left.\sum_{m=0}^{\infty} \mathrm{I}_{s 0}^{(2)} \mathcal{T}_{m_{r}}\right|_{r=R_{e}}\left(\mathcal{D}_{m 0}^{D} \overline{\mathrm{cs}}_{p m}+\mathcal{F}_{m 0}^{D} \overline{\mathrm{ss}}_{p m}\right) \\
& +\left.\sum_{l=1}^{\infty} \pi \mathcal{F}_{n l}^{D} \mathrm{I}_{s l}^{(3)} \delta_{p n} \mathcal{U}_{n l_{r}}\right|_{r=R_{e}}+\left.\sum_{m=0}^{\infty} \sum_{l=1}^{\infty} \mathrm{I}_{s l}^{(2)} \mathcal{U}_{m l_{r}}\right|_{r=R_{e}}\left(\mathcal{D}_{m l}^{D} \overline{\mathrm{Cs}}_{p m}+\mathcal{F}_{m l}^{D} \overline{\mathrm{Ss}}_{p m}\right)=0
\end{aligned}
$$

194

195

where $\overline{\mathrm{cc}}, \overline{\mathrm{ss}}, \overline{\mathrm{cs}}, \overline{\mathrm{sc}}$ are defined as follows

$$
\overline{\mathrm{cc}}_{p n}=\int_{\theta_{1}}^{\theta_{2}} \cos n \theta \cos p \theta \mathrm{d} \theta, \quad \overline{\mathrm{Ss}}_{p n}=\int_{\theta_{1}}^{\theta_{2}} \sin n \theta \sin p \theta \mathrm{d} \theta,
$$

$$
\overline{\mathrm{sc}}_{p n}=\int_{\theta_{1}}^{\theta_{2}} \sin n \theta \cos p \theta \mathrm{d} \theta, \quad \overline{\mathrm{Cs}}_{p n}=\int_{\theta_{1}}^{\theta_{2}} \cos n \theta \sin p \theta \mathrm{d} \theta .
$$

Summation of (46) and (50) plus summation of (49) and (51) gives an inhomogeneous linear system in the unknown coefficients $\mathcal{D}^{D}$ and $\mathcal{F}^{D}$. Once they are known, the coefficients $\mathcal{A}^{D}$ and $\mathcal{B}^{D}$ can be obtained through (38)(39), while $\mathcal{C}^{D}$ and $\mathcal{E}^{D}$ are given by (35).

\subsection{Radiation potential solution}

The problem is linear, hence the radiation velocity potential outside and inside the OWC can be assumed proportional to the pressure acting on the free surface $S_{i}$. The general solutions are similar to (31)-(32):

$$
\begin{gathered}
\phi_{e}^{R}=-\frac{\mathrm{i} p_{a}}{\rho \omega} \sum_{n=0}^{\infty}\left\{\frac{\cosh k_{0}(h+z)}{\cosh k_{0} h} \frac{H_{n}^{(1)}\left(k_{0} r\right)}{\left.H_{n_{r}}^{(1)}\left(k_{0} r\right)\right|_{r=R_{e}}}\left(\mathcal{A}_{n 0}^{R} \cos n \theta+\mathcal{B}_{n 0}^{R} \sin n \theta\right)\right. \\
\left.+\sum_{l=1}^{\infty} \frac{\cosh k_{l}(h+z)}{\cosh k_{l} h} \frac{K_{n}\left(\bar{k}_{l} r\right)}{\left.K_{n_{r}}\left(\bar{k}_{l} r\right)\right|_{r=R_{e}}}\left(\mathcal{A}_{n l}^{R} \cos n \theta+\mathcal{B}_{n l}^{R} \sin n \theta\right)\right\} \\
\phi_{i}^{R}=-\frac{\mathrm{i} p_{a}}{\rho \omega} \sum_{n=0}^{\infty}\left\{\frac{\cosh k_{0}(h+z)}{\cosh k_{0} h} \mathcal{T}_{n}\left(\mathcal{D}_{n 0}^{R} \cos n \theta+\mathcal{F}_{n 0}^{R} \sin n \theta\right)\right. \\
\left.+\sum_{l=1}^{\infty} \frac{\cosh k_{l}(h+z)}{\cosh k_{l} h} \mathcal{U}_{n l}\left(\mathcal{D}_{n l}^{R} \cos n \theta+\mathcal{F}_{n l}^{R} \sin n \theta\right)\right\}-\frac{\mathrm{i} p_{a}}{\rho \omega}
\end{gathered}
$$




$$
\begin{aligned}
& \frac{\left.2 \pi \delta_{p n} \mathrm{I}_{s 0}^{(3)} \mathcal{D}_{n 0}^{R} \mathcal{T}_{n_{r}}\right|_{r=R_{e}}}{\epsilon_{p}}+\left.\sum_{m=0}^{\infty} \mathrm{I}_{s 0}^{(2)} \mathcal{T}_{m_{r}}\right|_{r=R_{e}}\left(\mathcal{D}_{m 0}^{R} \overline{\mathrm{cc}}_{p m}+\mathcal{F}_{m 0}^{R} \overline{\mathrm{sc}}_{p m}\right) \\
& +\sum_{l=1}^{\infty} \frac{\left.2 \pi \mathcal{D}_{n l}^{R} \mathrm{I}_{s l}^{(3)} \delta_{p n} \mathcal{U}_{n l_{r}}\right|_{r=R_{e}}}{\epsilon_{p}}+\left.\sum_{m=0}^{\infty} \sum_{l=1}^{\infty} \mathrm{I}_{s l}^{(2)} \mathcal{U}_{m l_{r}}\right|_{r=R_{e}}\left(\mathcal{D}_{m l}^{R} \overline{\mathrm{cc}}_{p m}+\mathcal{F}_{m l}^{R} \overline{\mathrm{sc}}_{p m}\right)=0,
\end{aligned}
$$




$$
\begin{aligned}
& \left.\pi \delta_{p n} \mathrm{I}_{s 0}^{(3)} \mathcal{F}_{n 0}^{R} \mathcal{T}_{n_{r}}\right|_{r=R_{e}}+\left.\sum_{m=0}^{\infty} \mathrm{I}_{s 0}^{(2)} \mathcal{T}_{m_{r}}\right|_{r=R_{e}}\left(\mathcal{D}_{m 0}^{R} \overline{\mathrm{Cs}}_{p m}+\mathcal{F}_{m 0}^{R} \overline{\mathrm{ss}}_{p m}\right) \\
& +\left.\sum_{l=1}^{\infty} \pi \mathcal{F}_{n l}^{R} \mathrm{I}_{s l}^{(3)} \delta_{p n} \mathcal{U}_{n l_{r}}\right|_{r=R_{e}}+\left.\sum_{m=0}^{\infty} \sum_{l=1}^{\infty} \mathrm{I}_{s l}^{(2)} \mathcal{U}_{m l_{r}}\right|_{r=R_{e}}\left(\mathcal{D}_{m l}^{R} \overline{\mathrm{cs}}_{p m}+\mathcal{F}_{m l}^{R} \overline{\mathrm{ss}}_{p m}\right)=0 .
\end{aligned}
$$

where $K$ is an empirical coefficient depending on the turbine characteristics, $D$ is the outer diameter of the turbine rotor, $\rho_{a}$ the air density, $V_{0}$ the volume of air in the chamber when $\eta=0, c_{a}$ the speed of sound in air, while $q$ represents the complex part of $Q$ independent on time, i.e.

$$
Q=\operatorname{Re}\left\{q e^{-\mathrm{i} \omega t}\right\}=\operatorname{Re}\left\{e^{-\mathrm{i} \omega t} \int_{S_{i}} \frac{\partial \Phi}{\partial z} \mathrm{~d} S_{i}\right\}
$$

The flux $q$ can be further decomposed into radiation and diffraction components

$q=q^{D}+q^{R}, \quad q^{D}=\int_{S_{i}} \frac{\partial \phi_{i}^{D}}{\partial z} \mathrm{~d} S_{i}=\Gamma A, \quad q^{R}=\int_{S_{i}} \frac{\partial \phi_{i}^{R}}{\partial z} \mathrm{~d} S_{i}=-(\mathcal{B}-\mathrm{i} \mathcal{C}) p_{a}$, 
with $\Gamma$ being the complex exciting force, while the real quantities $\mathcal{B}$ and $\mathcal{C}$ represent respectively the radiation damping and the added mass due to the radiation wave field. The averaged power output over a wave period is

$$
\bar{P}_{\text {out }}=\frac{K D}{2 N \rho_{a}}\left|p_{a}\right|^{2}=\frac{A^{2}|\Gamma|^{2} K D}{2 N \rho_{a}\left[\left(\frac{K D}{N \rho_{a}}+\mathcal{B}\right)^{2}+\left(\frac{\omega V_{0}}{c_{a}^{2} \rho_{a}}+\mathcal{C}\right)^{2}\right]},
$$

hence the corresponding capture factor can be defined as the ratio between the generated power (65) and the energy influx of incident waves with amplitude $A$ per OWC width $2 R_{e}$ (Michele et al., 2016b)

$$
C_{F}=\frac{|\Gamma|^{2} K D}{2 \rho g R_{e} C_{g} N \rho_{a}\left[\left(\frac{K D}{N \rho_{a}}+\mathcal{B}\right)^{2}+\left(\frac{\omega V_{0}}{c_{a}^{2} \rho_{a}}+\mathcal{C}\right)^{2}\right]},
$$

where $C_{g}$ is the group velocity (Mei et al., 2005). Maximum efficiency of the capture factor (66) can be achieved if both the resonance condition, i.e. $\left(\mathcal{C}-\omega V_{0} / c_{a}^{2} \rho_{a}\right)=0$, and the identity $\mathcal{B}=K D / N \rho_{a}$ are satisfied. However, resonance is not always possible, mainly because of the difficulty in changing the structural parameter $V_{0} / c_{a}^{2} \rho_{a}$. In any case, if $\left(\mathcal{C}-\omega V_{0} / c_{a}^{2} \rho_{a}\right) \neq 0$, the optimal damping force exerted by the turbine can be chosen such that the derivative of $C_{F}$ (66) with respect to $K D / N \rho_{a}$ is zero. This condition holds if

$$
\frac{K D}{N \rho_{a}}=\sqrt{\mathcal{B}^{2}+\left(\frac{\omega V_{0}}{c_{a}^{2} \rho_{a}}+\mathcal{C}\right)^{2}} .
$$

Substitution of the latter expression in (66) yields the optimized capture factor $C_{F o p t}$

$$
C_{\text {Fopt }}=\frac{|\Gamma|^{2} \sqrt{\mathcal{B}^{2}+\left(\frac{\omega V_{0}}{c_{a}^{2} \rho_{a}}+\mathcal{C}\right)^{2}}}{2 \rho g R_{e} C_{g}\left[\left(\sqrt{\mathcal{B}^{2}+\left(\frac{\omega V_{0}}{c_{a}^{2} \rho_{a}}+\mathcal{C}\right)^{2}}+\mathcal{B}\right)^{2}+\left(\frac{\omega V_{0}}{c_{a}^{2} \rho_{a}}+\mathcal{C}\right)^{2}\right]} .
$$

If also resonance occurs, expression (68) yields the the maximum value of the capture factor $C_{F \max }$

$$
C_{F \max }=\frac{|\Gamma|^{2}}{8 \rho g R_{e} C_{g} \mathcal{B}} .
$$


Note that in the case of axisymmetric bodies, i.e. without the skirt, the latter relation yields after some algebra

$$
C_{F \max }=\frac{1}{2 k_{0} R_{e}}
$$

i.e. two times smaller than the maximum that can be reached by an oscillating flap-type wave surge converter in open sea having width equal to $2 R_{e}$ (Michele et al., 2016b). Now we derive several integral relations to perform a numerical check of the hydrodynamic quantities $\Gamma$ and $\mathcal{B}$. Applying Green's theorem to $\phi^{R}$ and its complex conjugate over the entire fluid domain $\Omega$ yields

$$
\frac{1}{\rho \omega} \operatorname{Re}\left\{\int_{S_{i}} p^{*} \phi_{i}^{R} \mathrm{~d} S_{i}\right\}=\operatorname{Re}\left\{\int_{S_{\infty}}-\mathrm{i} \phi_{e}^{R} \phi_{e}^{R^{*}} \mathrm{~d} S_{\infty}\right\},
$$

where $(\cdot)^{*}$ denotes the complex conjugate of $(\cdot)$ and $S_{\infty}$ is a vertical cylinder of large radius $r \rightarrow \infty$ and height $h$. The radiation potential $\phi_{e}^{R}$ in the far field can be approximated by

$$
\phi_{e}^{R} \sim \frac{\mathcal{A}(\theta)}{\sqrt{k_{0} r}} e^{\mathrm{i} k_{0} r} \cosh k_{0}(h+z)
$$

in which $\mathcal{A}(\theta)$ represents the angular variation of the radiated waves at large distances. From (54) we get

$$
\mathcal{A}(\theta)=-\frac{\mathrm{i} p_{a}}{\rho \omega \cosh k_{0} h} \sqrt{\frac{2}{\pi}} \sum_{n=0}^{\infty} \frac{e^{-\mathrm{i}(\pi / 4+n \pi / 2)}\left(\mathcal{A}_{n 0}^{R} \cos n \theta+\mathcal{B}_{n 0}^{R} \sin n \theta\right)}{\left.H_{n_{r}}^{(1)}\left(k_{0} r\right)\right|_{r=R_{e}}} .
$$

Substitution of (64), (72) and (73) into the integrals (71) yields after some algebra

$$
\begin{aligned}
\mathcal{B} & =\frac{\rho \omega C_{0}}{\left|p_{a}\right|^{2}} \int_{0}^{2 \pi}|\mathcal{A}(\theta)|^{2} \mathrm{~d} \theta \\
& =\frac{2 D_{0}}{\rho \omega \cosh ^{2} k_{0} h}\left(\frac{2\left|\mathcal{A}_{00}^{R}\right|^{2}}{\left.H_{0_{r}}^{(1)}\left(k_{0} r\right)\right|_{r=R_{e}}}+\sum_{n=1}^{\infty} \frac{\left|\mathcal{A}_{n 0}^{R}\right|^{2}+\left|\mathcal{B}_{n 0}^{R}\right|^{2}}{\left.H_{n_{r}}^{(1)}\left(k_{0} r\right)\right|_{r=R_{e}}}\right),
\end{aligned}
$$

where

$$
C_{0}=\int_{-h}^{0} \cosh ^{2} k_{0}(h+z) \mathrm{d} z=\frac{2 k_{0} h+\sinh 2 k_{0} h}{4 k_{0}} .
$$


Expression (74) relates the radiation damping and the amplitude of the radiated waves at large distances and can be used for numerical check purposes. Similarly, applying Green's theorem to the radiation and diffraction velocity potentials gives

$$
\frac{\mathrm{i} p}{\rho \omega} \int_{S_{i}} \phi_{i_{z}}^{D} \mathrm{~d} S_{i}=-\int_{S_{\infty}}\left(\phi^{I} \phi_{e_{r}}^{R}-\phi_{e} \phi_{r}^{I}\right) \mathrm{d} S_{\infty},
$$

where $\phi^{I}$ is the velocity potential of the incident waves with amplitude $A$ and frequency $\omega$ directed along the $x$-axis

$$
\phi^{I}=-\frac{\mathrm{i} A g}{\omega} \frac{\cosh k_{0}(h+z)}{\cosh k_{0} h} e^{\mathrm{i} k_{0} r \cos \theta} .
$$

By the method of the stationary phase, after a long but straightforward algebra we obtain

$$
\Gamma=\frac{4 g C_{0}}{\omega \cosh ^{2} k_{0} h} \sum_{n=0}^{\infty} \frac{\mathcal{A}_{n 0}^{R} e^{-\mathrm{i} n \pi / 2} \cos n \pi}{\left.H_{n_{r}}^{(1)}\left(k_{0} r\right)\right|_{r=R_{e}}} .
$$

This is the Haskind-Hanaoka formula for the OWC in open sea and relates the exciting force $\Gamma$ with the amplitude of the radiated waves in the direction opposite to the incoming waves $\theta=\pi$. The latter relation is used in the next section to check numerical evaluations for the radiation and diffraction velocity potentials.

\subsection{Results and discussion}

In this section we examine the effects of the OWC geometry and turbine characteristics on the hydrodynamic behaviour and energy extraction efficiency. For the sake of example, let us consider the following fixed parameters: $A=1 \mathrm{~m}, h=10 \mathrm{~m}, \rho=1000 \mathrm{~kg} \mathrm{~m}^{-3}, \rho_{a}=1 \mathrm{~kg} \mathrm{~m}^{-3}$ and $c_{a}=340 \mathrm{~m}$ $\mathrm{s}^{-1}$. Since in the expressions for the velocity potentials (31)-(32)-(54)-(55) there are infinite terms, we need to truncate the summations up to a limiting value $n=N$ and $l=L$ for practical computations. In this work we use $N=L=100$ to achieve a precision of 2 decimal places (Deng et al., 2013).

\subsubsection{Effects of the skirt height}

Here we examine the effects of the skirt height $h_{s}$ on the exciting force $\Gamma$, radiation damping $\mathcal{B}$ and added mass $\mathcal{C}$. Let us fix the following parameters 
$R_{e}=h / 2, \theta_{1}=5 \pi / 4 \mathrm{rad}, \theta_{2}=3 \pi / 4 \mathrm{rad}$ and $h_{c}=0.2 \times h$. The latter numerical values are the same adopted in Deng et al. (2013), thus we can perform several validations of our numerical results. As in Deng et al. (2013), let us define the non-dimensional hydrodynamic quantities

$$
\tilde{\Gamma}=\Gamma \frac{\sqrt{g / h}}{g h}, \quad \tilde{\mathcal{B}}=\mathcal{B} \frac{\rho \sqrt{g / h}}{g}, \quad \tilde{\mathcal{C}}=\mathcal{C} \frac{\rho \sqrt{g / h}}{g},
$$

and take as a first case $R_{i}=0$. Figures 2(a)-2(c) shows the behaviour of $\tilde{\Gamma}$, $\tilde{\mathcal{B}}$ and $\tilde{\mathcal{C}}$ versus the non-dimensional frequency of the incident waves $\omega^{2} h / g$ for five different configurations. Each configuration has a specific value of the skirt height, that varies from $h-h_{c}$ to zero. The limiting value in which $h_{s}=h-h_{c}$, corresponds to the case of an OWC supported by a coaxial tube-sector-shaped structure analysed by Deng et al. (2013), while the case $h_{s}=0$ corresponds to the model developed by Evans and Porter (1997). Excellent agreement with the numerical results of Deng et al. (2013) (Fig. 4 and Fig. 5, case $d / h=0.2)$ is obtained. This test validates the effectiveness of the method of solution adopted in this work for the novel device.

As in Garrett (1970), Evans and Porter (1997) and Deng et al. (2013), resonant interactions of the heave and sloshing modes inside the chamber occur. This is the reason why each peak for each hydrodynamic parameter is localized at the same frequency. Since $R_{i}=0$, the resonances appear approximately at zeros of the Bessel function $J_{n}^{\prime}\left(k_{0} R_{e}\right)$ satisfying the boundary condition for the sloshing modes inside a vertical cylinder of radius $R_{e}$ and height $h$.

Note that the OWC without the skirt does not excite the sloshing modes proportional to $\cos \theta$ or $\sin \theta$. In this case, the first sloshing resonance satisfies the second zero of $J_{0}^{\prime}\left(k_{0} R_{e}\right)$ which occurs for $k_{0} R_{e} \simeq 3.831$. Similar results are obtained in Evans and Porter (1997). On the contrary, if $h_{s} \neq 0$, we obtain two additional peaks between the Helmholtz mode and the frequency corresponding to $k_{0} R_{e} \simeq 3.831$. These peaks are related to the firsts roots of $J_{1}^{\prime}\left(k_{0} R_{e}\right)$ and $J_{2}^{\prime}\left(k_{0} R_{e}\right)$.

As the height $h_{s}$ increases, the first resonant peak of $\tilde{\Gamma}, \tilde{\mathcal{B}}$ and $\tilde{\mathcal{C}}$ related to the Helmholtz mode moves towards small frequencies. In particular, in the case of $\tilde{\Gamma}$ shown in figure $2($ a), the first peak tends to become higher and sharper as well. On the other hand, the peaks related to the sloshing modes seem to be unaffected by $h_{s}$.

The optimal capture factor $C_{F o p t}(68)$ is shown in figure $2(\mathrm{~d})$. Within this range of $\omega$, except for the case without the skirt, four modes are excited 


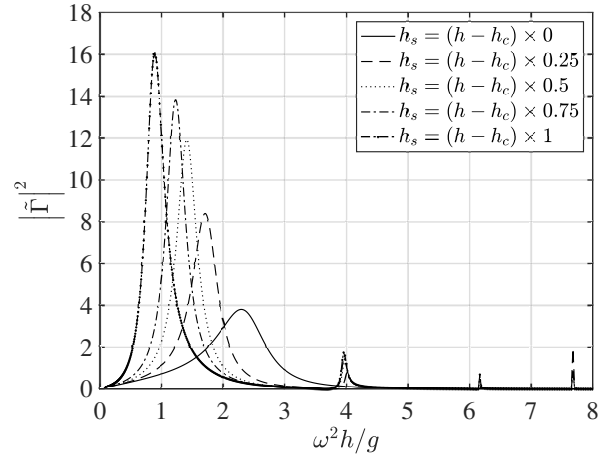

(a)

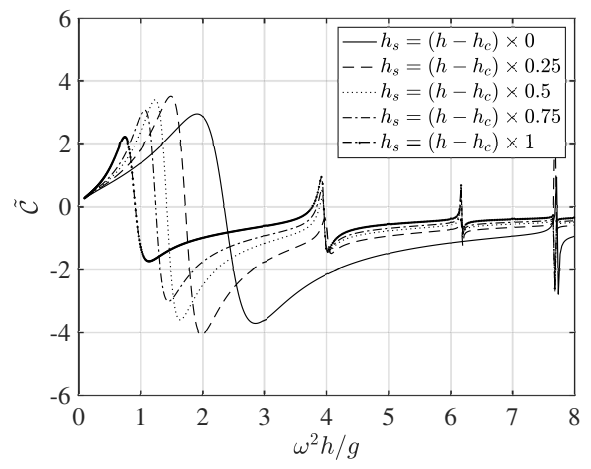

(c)

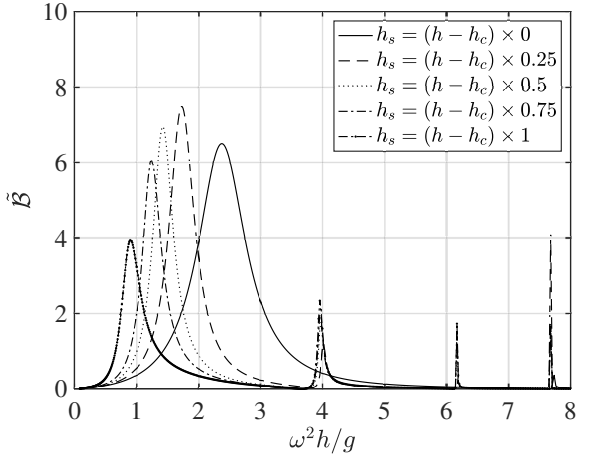

(b)

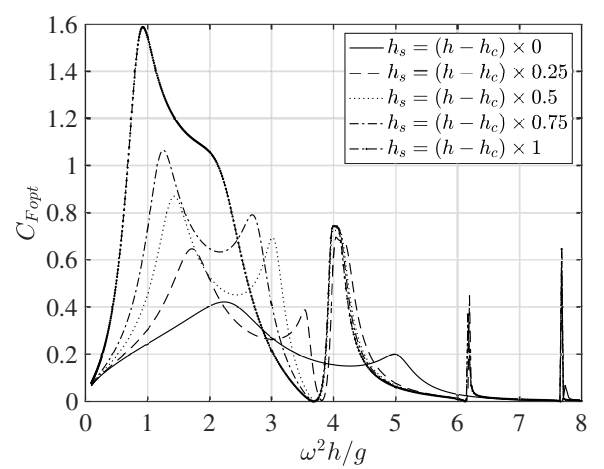

(d)

Figure 2: The effects of the skirt height $h_{s}$ on the hydrodynamic behaviour. 2(a) nondimensional exciting force $|\tilde{\Gamma}|^{2}, 2(\mathrm{~b})$ non-dimensional radiation damping $\tilde{\mathcal{B}}, 2(\mathrm{c})$ nondimensional added mass $\tilde{\mathcal{C}}$ and $2(\mathrm{~d})$ optimal capture factor $C_{F o p t}$ of each configuration versus non-dimensional incident wave frequency $\omega^{2} h / g$. 
hence four maxima occur for $C_{F o p t}$. The same figure shows that the first resonant peak is the widest and sometimes, depending on $h_{s}$, the largest. Values of $C_{F o p t}$ can be larger than 1, i.e. larger than the maximum of a bi-dimensional absorber in a channel flume (Mei et al., 2005). Note that the most efficient configuration corresponds to the case of a skirt extending to the bottom. Next, let the internal radius be $R_{i}=0.75 \times R_{e}$ and evaluate $\tilde{\Gamma}, \tilde{\mathcal{B}}, \tilde{\mathcal{C}}$ and $C_{F o p t}$ for the same configurations analysed before. Now the internal radius differs from zero, thus the resonant peaks are associated with the sloshing modes of an isolated annular cylinder with fluid occupying the volume $\Omega_{i}$. Since the general solution of the velocity potential includes both $J_{n}$ and $Y_{n}$ the corresponding wave-number $k_{0}$ for each sloshing mode must satisfy the following eigenvalue condition

$$
J_{n}\left(k_{0} R_{i}\right) Y_{n}\left(k_{0} R_{e}\right)-J_{n}\left(k_{0} R_{e}\right) Y_{n}\left(k_{0} R_{i}\right)=0 .
$$

Figure 3 shows that four peaks are present in the computed range of frequencies. Maxima of all the resonant peaks are almost unaffected, while the peaks corresponding to the sloshing modes tend to move towards smaller frequencies. Note also that the second and third peaks for $C_{F o p t}$ increase their width, hence in this case the presence of an internal radius has benefits in terms of power extraction efficiency.

\subsubsection{Effects of the skirt opening}

Now we analyse the effects of the skirt opening $\theta_{2}-\theta_{1}$ on the same hydrodynamic parameters analysed in the previous section $\Gamma, \mathcal{B}$ and $\mathcal{C}$. Let the external radius be $R_{e}=h / 2$ and fix both skirt height $h_{s}=0.5 \times\left(h-h_{c}\right)$ and internal radius $R_{i}=0$. Five skirt opening angles have been analysed, respectively described by $\theta_{1}=\theta_{2}=\pi, \theta_{1}=5 \pi / 4$ and $\theta_{2}=3 \pi / 4, \theta_{1}=3 \pi / 2$ and $\theta_{2}=\pi / 2, \theta_{1}=7 \pi / 4$ and $\theta_{2}=\pi / 4, \theta_{1}=2 \pi$ and $\theta_{2}=0 \mathrm{rad}$.

Figure 4 shows the effects of the opening angle for different incident wave non-dimensional frequencies on the hydrodynamic parameters $\Gamma, \mathcal{B}$ and $\mathcal{C}$ and the optimal capture factor $C_{F o p t}$. As shown by figures $4(\mathrm{a})-4(\mathrm{c})$, when the opening increases, the Helmholtz mode resonant peaks decrease while the corresponding resonant frequencies increase. This is less visible for the sloshing modes whose position is almost unvaried. We shall point out that similar results are obtained by Deng et al. (2013) for a skirt extending from the OWC to the sea bottom.

Figure $4(\mathrm{~d})$ shows the behaviour of the optimal capture factor $C_{F o p t}$. The best configuration with larger and wider peaks corresponds to the symmetric 


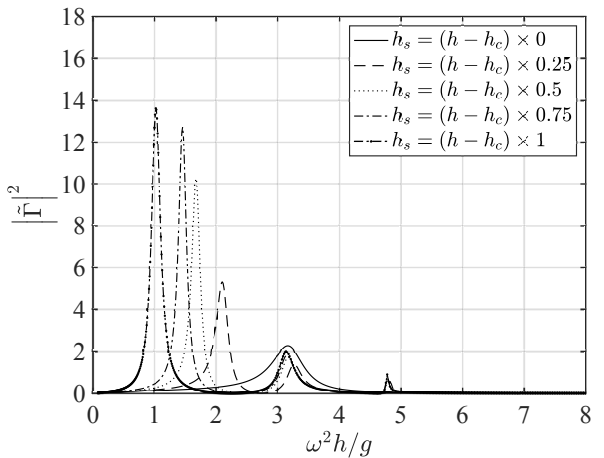

(a)

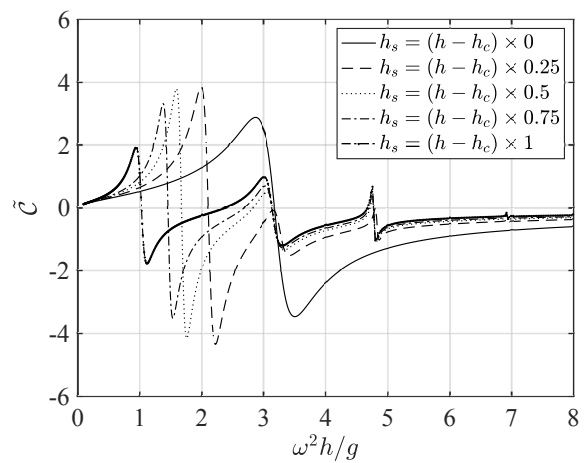

(c)

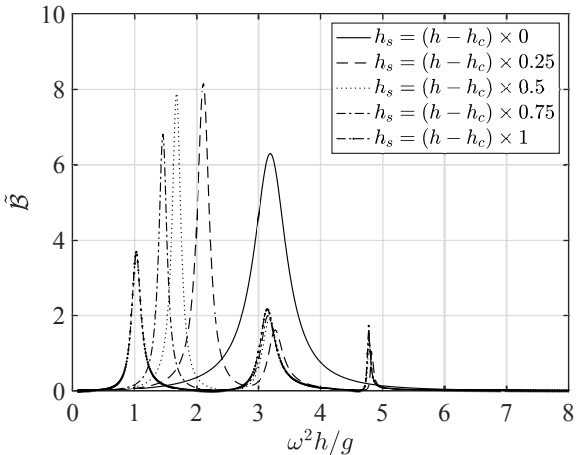

(b)

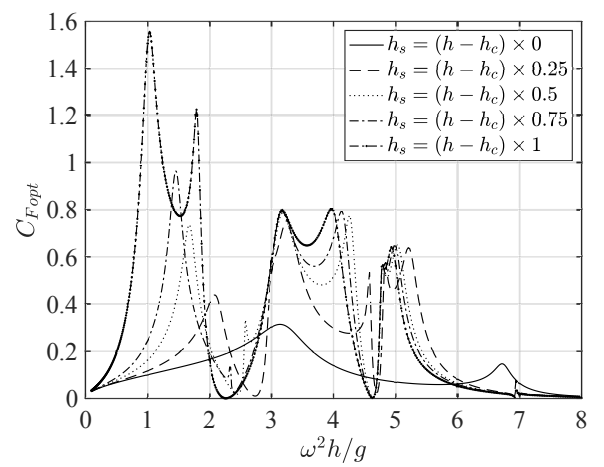

(d)

Figure 3: The effects of skirt height $h_{s}$ and internal radius $R_{i}$ on the hydrodynamic behaviour. 3(a) non-dimensional exciting force $|\tilde{\Gamma}|^{2}, 3(\mathrm{~b})$ non-dimensional radiation damping $\tilde{\mathcal{B}}, 3(\mathrm{c})$ non-dimensional added mass $\tilde{\mathcal{C}}$ and $3(\mathrm{~d})$ capture factor $C_{F o p t}$ of each configuration versus non-dimensional incident wave frequency $\omega^{2} h / g$. The value of the internal radius corresponds to $R_{i}=0.75 \times R_{e}$. 
case $\theta_{1}=3 \pi / 2$ and $\theta_{2}=\pi / 2$ with opening angle equal to $\pi \mathrm{rad}$. This result suggests that the skirt plays an important role on the power extraction efficiency, however one should take care of its effects on the OWC structural resistance that could penalise the overall behaviour and durability in real seas. Now we change the internal radius to $R_{i}=0.75 \times R_{e}$. Figures 5(a)$5(\mathrm{~d})$ show $\tilde{\Gamma}, \tilde{\mathcal{B}}, \tilde{\mathcal{C}}$ and $C_{F o p t}$ versus $\omega^{2} h / g$. The same considerations of the previous section can be extended here, i.e. the maximum values of the resonant peaks almost preserve their values, while the peaks of the sloshing modes become wider.

\section{Power extraction efficiency in random waves}

In this section we investigate the effects of random waves on the generated power. Without loss of generality, we can adopt the JONSWAP spectrum $S_{\zeta}$ to describe the incident wave field (Goda, 2000)

$$
S_{\zeta}(\omega)=\frac{\alpha H_{s}^{2}}{\omega}\left(\frac{\omega_{p}}{\omega}\right)^{4} \exp \left[-1,25\left(\frac{\omega_{p}}{\omega}\right)^{4}\right] \gamma^{\exp \left[-\left(\omega / \omega_{p}-1\right)^{2} /(2 \sigma)\right]},
$$

in which $H_{s}$ is the significant wave height, $\omega_{p}$ denotes the peak frequency and

$$
\alpha=\frac{0.0624(1.094-0.01915 \ln \gamma)}{0.23+0.0336 \gamma-0.185(1,9+\gamma)^{-1}}, \sigma=\left\{\begin{array}{l}
0.07: \omega \leq \omega_{p} \\
0.09: \quad \omega>\omega_{p}
\end{array}, \gamma=3.3\right.
$$

Because of linearity, the pressure oscillation inside the OWC can be written as

$$
P_{a}\left(t, \omega_{p}\right)=\sum_{n=1}^{\infty} \sqrt{2 S_{\zeta}\left(\omega_{n}\right) \Delta \omega} \operatorname{RAO}\left(\omega_{n}\right) \cos \left(\omega_{n} t+\delta_{n}\right),
$$

where $\omega_{n}$ is the $n$th component of the discretised spectrum, $\Delta \omega$ is the frequency step, $\delta_{n}$ is a random phase related to $\omega_{n}$ while the term RAO is the response amplitude operator for the air pressure $p_{a}$, i.e.

$$
\operatorname{RAO}\left(\omega_{n}\right)=\left|\frac{\Gamma\left(\omega_{n}\right)}{\left(\frac{K D}{N \rho_{a}}+\mathcal{B}\left(\omega_{n}\right)\right)-\mathrm{i}\left(\frac{\omega_{n} V_{0}}{c_{a}^{2} \rho_{a}}+\mathcal{C}\left(\omega_{n}\right)\right)}\right| .
$$




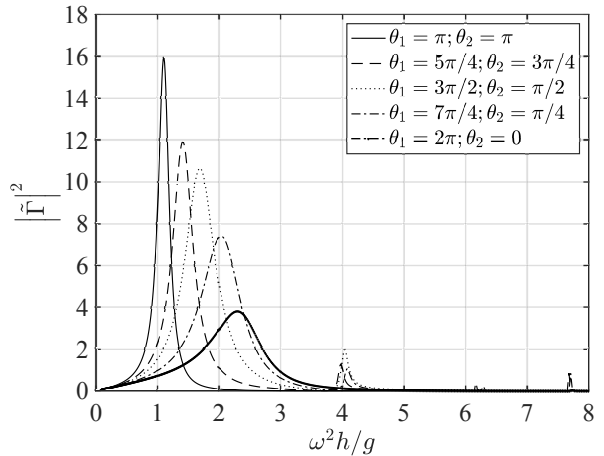

(a)

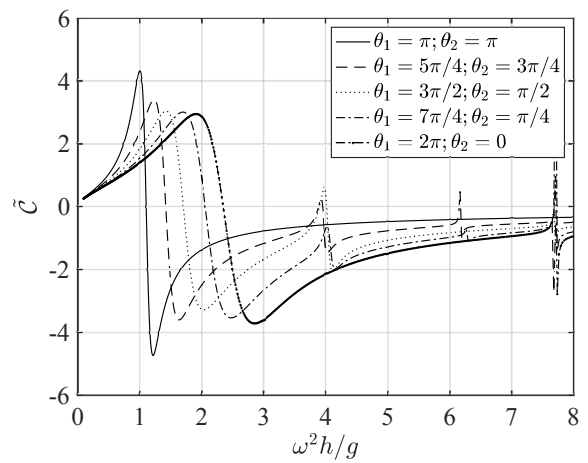

(c)

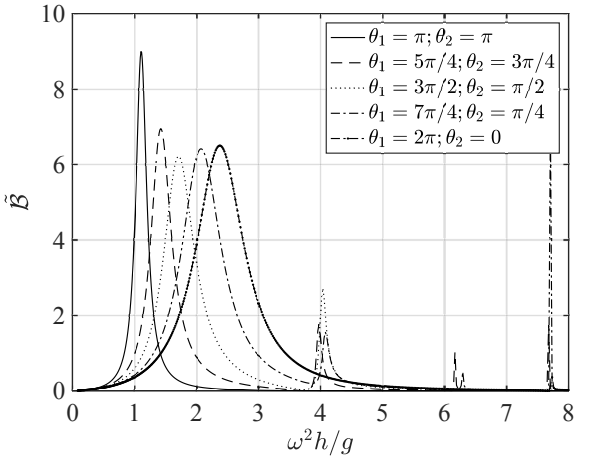

(b)

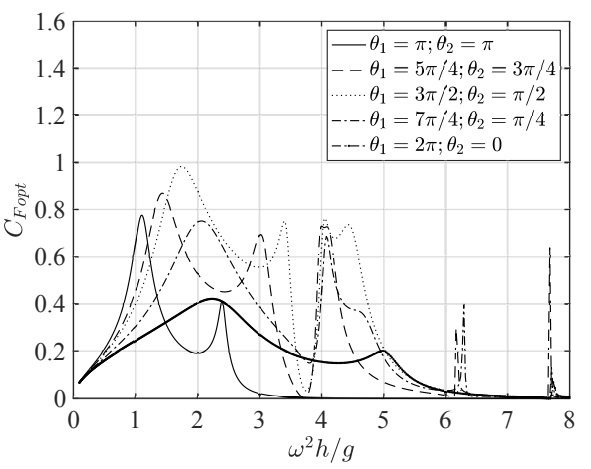

(d)

Figure 4: The effects of the skirt opening $\theta_{2}-\theta_{1}$. 4(a) non-dimensional exciting force $|\tilde{\Gamma}|^{2}, 4(\mathrm{~b})$ non-dimensional radiation damping $\tilde{\mathcal{B}}, 4(\mathrm{c})$ non-dimensional added mass $\tilde{\mathcal{C}}$ and 4(d) optimized capture factor $C_{F o p t}$ of each configuration versus non-dimensional incident wave frequency $\omega^{2} h / g$. 


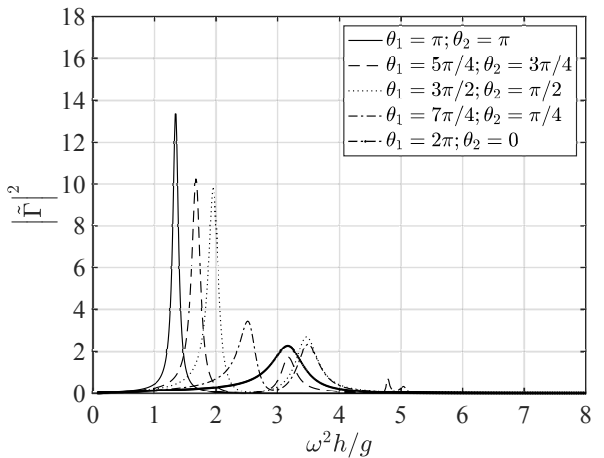

(a)

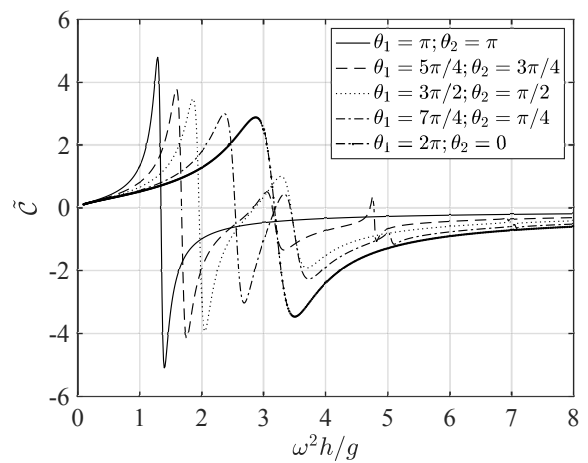

(c)

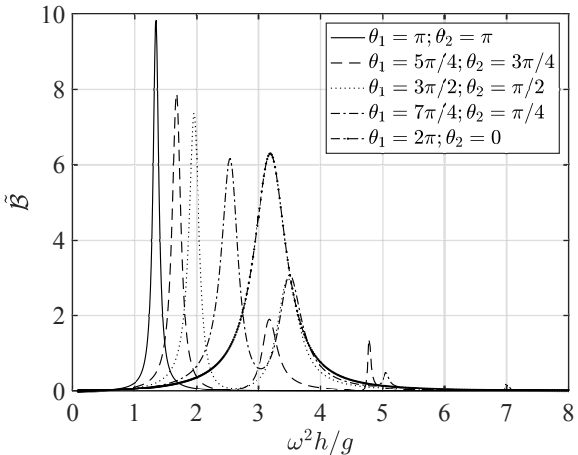

(b)

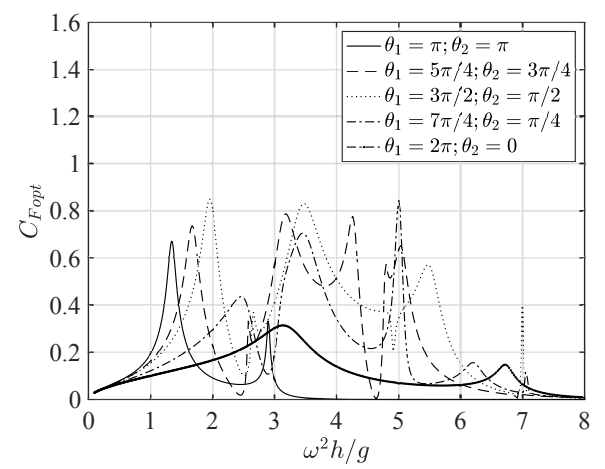

(d)

Figure 5: The effects of the skirt opening $\theta_{2}-\theta_{1}$ and internal radius $R_{i}$ on the hydrodynamic behaviour. 5(a) non-dimensional exciting force $|\tilde{\Gamma}|^{2}, 5(\mathrm{~b})$ non-dimensional radiation damping $\tilde{\mathcal{B}}, 5(\mathrm{c})$ non-dimensional added mass $\tilde{\mathcal{C}}$ and $5(\mathrm{~d})$ optimized capture factor $C_{F o p t}$ of each configuration versus non-dimensional incident wave frequency $\omega^{2} h / g$. The value of the internal radius corresponds to $R_{i}=0.75 \times R_{e}$. 
377

Then, the instantaneous generated power is

$$
\begin{aligned}
P_{s}\left(t, \omega_{p}\right) & =\frac{K D}{N}\left[\sum_{n=1}^{\infty} \sqrt{2 S_{\zeta}\left(\omega_{n}\right) \Delta \omega} \operatorname{RAO}\left(\omega_{n}\right) \cos \left(\omega_{n} t+\delta_{n}\right)\right]^{2} \\
& -\frac{V}{c_{a}^{2}} \sum_{n=1}^{\infty} \sqrt{2 S_{\zeta}\left(\omega_{n}\right) \Delta \omega} \operatorname{RAO}\left(\omega_{n}\right) \cos \left(\omega_{n} t+\delta_{n}\right) \\
& \times \sum_{n=1}^{\infty} \sqrt{2 S_{\zeta}\left(\omega_{n}\right) \Delta \omega} \operatorname{RAO}\left(\omega_{n}\right) \omega_{n} \sin \left(\omega_{n} t+\delta_{n}\right) .
\end{aligned}
$$

From the foregoing expression we obtain the averaged generated power (Michele et al., 2016b)

$$
\bar{P}_{s}\left(\omega_{p}\right)=\lim _{\tau \rightarrow \infty} \frac{1}{\tau} \int_{0}^{\tau} P_{s} \mathrm{~d} t=\frac{K D}{N} \sum_{n=1}^{\infty} S_{\zeta}\left(\omega_{n}\right) \Delta \omega \mathrm{RAO}^{2}\left(\omega_{n}\right),
$$

whose expression in the limit $\Delta \omega \rightarrow 0$ becomes

$$
\bar{P}_{s}\left(\omega_{p}\right)=\frac{K D}{N} \int_{0}^{\infty} S_{\zeta}(\omega) \operatorname{RAO}^{2}(\omega) \mathrm{d} \omega .
$$

Defining $P_{\zeta}$ as the total incident wave power per unit crest width

$$
P_{\zeta}\left(\omega_{p}\right)=\int_{0}^{\infty} \rho g C_{g}(\omega) S_{\zeta}(\omega) \mathrm{d} \omega,
$$

the capture width ratio in random seas $C_{F \zeta}$ can then be written as

$$
C_{F \zeta}\left(\omega_{p}\right)=\frac{\bar{P}_{s}}{2 R_{e} P_{\zeta}} .
$$

Let us compare a fixed configuration when excited by random and monochromatic waves. Here we assume $A=1 \mathrm{~m}, h=10 \mathrm{~m}, \rho=1000 \mathrm{~kg} \mathrm{~m}^{-3}, \rho_{a}=1$ $\mathrm{kg} \mathrm{m}{ }^{-3}, c_{a}=340 \mathrm{~m} \mathrm{~s}^{-1}$, the external radius $R_{e}=h / 2$ and two values of the internal radius, $R_{i}=0$ and $R_{i}=0.75 \times R_{e}$, respectively. Let us fix the optimal value of $K D / N \rho_{a}$ that maximizes power extraction for the fixed frequency $\omega=1 \mathrm{rad} \mathrm{s}^{-1}$ and assume the symmetric configuration $\theta_{1}=3 \pi / 2$ and $\theta_{2}=\pi / 2$ rad maximizing power extraction efficiency. In other words, we have fixed both OWC geometry and turbine characteristics and optimized 
them for a frequency representing the wave climate of a particular area. This situation can be of practical interest because of the difficulty in tuning the turbine speed/geometry with a wide range of incident wave frequencies (López et al., 2014).

Figure 6(a) shows the behaviour of $C_{F \zeta}$ and $C_{F}$ for the case with null internal radius $R_{i}=0$. The abscissa for $C_{F \zeta}$ refers to the peak frequency $\omega_{p}$ of the JONSWAP spectrum, while the abscissa for $C_{F}$ refers to the frequency of the monochromatic incident waves. In both cases the maxima of $C_{F \zeta}$ are smaller than the resonant peaks of $C_{F}$, while the system becomes more efficient outside the resonant frequencies. Furthermore, the narrow peak related to the resonance of the first sloshing mode decreases significantly and reduces to a small hump. This fact is consistent with the bad coupling between the incident wave spectrum and the natural modes characterized by small radiation damping. Similar results are obtained in the context of flap-type oscillating wave surge converters by Michele et al. (2016a), Michele et al. (2016b) and Sarkar et al. (2014).

Figure $6(\mathrm{~b})$ shows $C_{F \zeta}$ and $C_{F}$ respectively versus $\omega_{p}$ and $\omega$ for the second configuration with $R_{i}=0.75 \times R_{e}$. As before, we optimize $K D / N \rho_{a}$ for the fixed frequency $\omega=1 \mathrm{rad} \mathrm{s}^{-1}$. Also in this case the maxima of $C_{F \zeta}$ are smaller than those of $C_{F}$ and the spreading effect of the spectrum is evident. Differently, in the case shown in figure 6(a) the sloshing mode has a significant contribution because of the small sharpness of the resonant peak in $C_{F}$.

\section{Theoretical and experimental comparisons}

The damping force exerted by the turbine is usually modelled by an orifice above the OWC (Perez-Collazo et al., 2018b). In this case, in which an impulse turbine is used, the relation between the airflow $Q$ through the orifice and the air pressure $P$ inside the OWC chamber is quadratic (López et al., 2016), hence the linear relation used to model Wells turbines (62) fails and cannot be used here. Applying Bernoulli's theorem in correspondence of the orifice cross section we obtain

$$
P_{a}=\rho_{a} C_{q}^{2} \frac{Q|Q|}{2 \Omega_{o}},
$$

where $C_{q} \simeq 0.6$ is the dimensionless coefficient of discharge depending on the orifice geometry and $\Omega_{o}$ is the area of the orifice. Substitution of the latter 


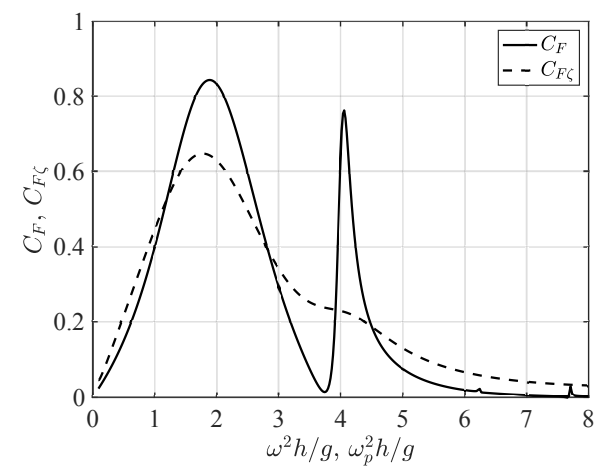

(a)

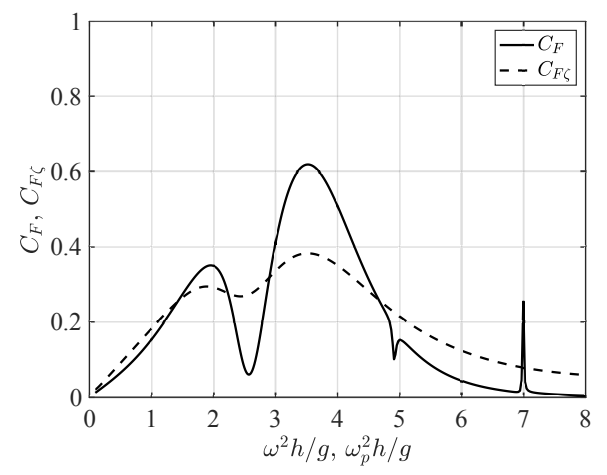

(b)

Figure 6: Comparison between the capture factor in random waves $C_{F \zeta}$ and the capture factor for monochromatic incident waves $C_{F}$ respectively versus peak spectral frequency $\omega_{p}$ and incident frequency $\omega$. Figure 6 (a) refers to the configuration with null internal radius $R_{i}$ while figure $6(\mathrm{~b})$ is related to the case with $R_{i}=0.75 \times R_{i}$. The turbine characteristics are optimized for the frequency $\omega=1 \mathrm{rad} \mathrm{s}^{-1}$.

expression in the nonlinear mixed boundary condition on the free surface yields (Mei et al., 2005):

$\Phi_{t t}+g \Phi_{z}+|\nabla \Phi|_{t}^{2}+\frac{1}{2} \nabla \Phi \cdot \nabla|\nabla \Phi|^{2}=-\frac{\rho_{a} C_{q}^{2}}{2 \rho \Omega_{o}^{2}}\left[\int_{S_{i}} \Phi_{z} \mathrm{~d} S_{i}\left|\int_{S_{i}} \Phi_{z} \mathrm{~d} S_{i}\right|\right]_{t}$, on $S_{i}$.

Now, by introducing the following non-dimensional quantities denoted by primes (Michele et al., 2018, 2019a; Michele and Renzi, 2019; Sammarco et al., 1997a,b):

$$
\begin{aligned}
& \left(x^{\prime}, y^{\prime}, z^{\prime}\right)=(x, y, z) / \lambda, \quad \Phi^{\prime}=\Phi /(A \omega \lambda), \quad t^{\prime}=t \omega, \\
& G=g /\left(\omega^{2} \lambda\right), \quad \epsilon=A / \lambda,
\end{aligned}
$$

expression (91) becomes

$$
\begin{aligned}
\Phi_{t^{\prime} t^{\prime}}^{\prime}+G \Phi_{z^{\prime}}^{\prime} & =\epsilon\left|\nabla^{\prime} \Phi^{\prime}\right|_{t^{\prime}}^{2}+\epsilon^{2} \frac{1}{2} \nabla^{\prime} \Phi^{\prime} \cdot \nabla^{\prime}\left|\nabla^{\prime} \Phi^{\prime}\right|^{2} \\
& -\epsilon \frac{\rho_{a} C_{q}^{2}}{2 \rho \Omega_{o}^{2}}\left[\int_{S_{i}} \Phi_{z^{\prime}}^{\prime} \mathrm{d} S_{i}\left|\int_{S_{i}} \Phi_{z^{\prime}}^{\prime} \mathrm{d} S_{i}\right|\right]_{t^{\prime}}, \text { on } S_{i},
\end{aligned}
$$

thus, if the wave steepness is small, i.e. $\epsilon \ll 1$, and the ratio between the area $S_{i}$ and the area of the orifice $\Omega_{o}$ is of order $O\left(1 / \epsilon^{2}\right)$, the nonlinear terms on 
the right hand side of (93) become small and weak if compared to the linear part on the left hand side. Applying the standard perturbation expansion technique to the velocity potential

$$
\Phi^{\prime}=\Phi_{1}^{\prime}+\epsilon \Phi_{2}^{\prime}+O\left(\epsilon^{2}\right),
$$

gives the condition (93) homogeneous and unforced at the leading order $O(1)$ :

$$
\Phi_{1_{t^{\prime} t^{\prime}}}^{\prime}+G \Phi_{1_{z^{\prime}}}^{\prime}=0, \quad \text { on } S_{i} .
$$

If we now return in physical variables and assume both harmonic motion and incident waves at $O(1)$, equation (95) becomes identical to the boundary condition on $S_{i}$ for $\phi_{i}^{D}(26)$, hence the solution of the velocity potential $\Phi_{1}$ corresponds to the diffraction velocity potential already found in Section 2.1. As a consequence, the air pressure inside the chamber at the leading order can be approximated by the following expression

$$
P_{a}=\rho_{a} C_{q}^{2} \frac{\operatorname{Re}\left\{q^{D} e^{-\mathrm{i} \omega t}\right\}\left|\operatorname{Re}\left\{q^{D} e^{-\mathrm{i} \omega t}\right\}\right|}{2 \Omega_{o}^{2}} .
$$

The latter expression yields the averaged rate of work done by the air pressure inside the chamber

$$
\bar{P}_{\text {out }}=2 \rho_{a} C_{q}^{2} \frac{\left|q^{D}\right|^{3}}{3 \pi \Omega_{o}^{2}},
$$

and the corresponding capture factor

$$
C_{F \exp }=\frac{2 \rho_{a} C_{q}^{2}\left|q^{D}\right|^{3}}{3 \pi \Omega_{o}^{2} R_{e} A^{2} \rho g C_{g}} .
$$

\section{In order to validate the theory, comparisons are made with the experimental results of Perez-Collazo et al. (2018b). Channel flume and OWC character- istics are fixed and listed in Table 1. \\ 5.1. Monochromatic waves}

Figure 7 shows the values of the capture factor $C_{F e x p}$ versus the wave period $T$ in prototype values for both the analytical (expression (98)) and experimental model (see figure 10 in Perez-Collazo et al. (2018b)). In particular, figure $7(\mathrm{a})$ and figure $7(\mathrm{~b})$ refer to the different orifice diameters $d_{0}=0.015 \mathrm{~m}$ and $d_{0}=0.019 \mathrm{~m}$, respectively. The amplitude of the incident 
Table 1: Channel and OWC characteristics

\begin{tabular}{lll}
\hline Parameters & Symbol & Dimensions \\
\hline Depth & $h$ & $1 \mathrm{~m}$ \\
External radius & $R_{e}$ & $0.08 \mathrm{~m}$ \\
Internal radius & $R_{i}$ & $0 \mathrm{~m}$ \\
OWC draft & $h_{c}$ & $0.076 \mathrm{~m}$ \\
Skirt height & $h_{s}$ & $0.04 \mathrm{~m}$ \\
Skirt angle 1 & $\theta_{1}$ & $3 \pi / 2 \mathrm{rad}$ \\
Skirt angle 2 & $\theta_{2}$ & $\pi / 2 \mathrm{rad}$ \\
\hline
\end{tabular}

regular waves is $A=1 \mathrm{~m}$. The agreement between both models is good at large periods, however, for the case shown in figure 7(a) the theoretical capture factor is clearly overestimated when $T \in[7,8] \mathrm{s}$. This is a consequence of the Helmholtz-mode resonance around $T=5.5 \mathrm{~s}$. In this range of periods, nonlinearities, viscous dissipation and effects due to vortex shedding at the lower edges (Xu et al., 2016; Xu and Huang, 2019) are not weak anymore and become important. Moreover, the smaller the value of $d_{0}$, the greater the differences between the models. This is because the ratio $S_{i} / \Omega_{o}$ increases and strengthens the order of magnitude of the last term on the right-hand side of (93).

\subsection{Random waves}

In this section we analyse the amplitude response of the free surface elevation inside the air chamber in irregular wave conditions. Within the framework of a linearised theory we can write the spectrum of the averaged amplitude response as (Michele et al., 2016a):

$$
S_{\bar{\eta}}=\sqrt{2|\bar{\eta}|^{2} S_{\zeta} \Delta \omega}
$$

where $\bar{\eta}$ represents the averaged free-surface amplitude response inside the OWC chamber in monochromatic waves with $A=1 \mathrm{~m}$.

For the sake of example, let us consider the configuration with orifice diameter $d_{0}=0.015 \mathrm{~m}$, significant wave height $H_{s}=3.5 \mathrm{~m}$ and peak period $T_{p}=13.3 \mathrm{~s}$ in prototype values (Series C07 in Perez-Collazo et al. (2018b)). Figure 8 shows the theoretical and experimental spectra of the averaged amplitude response $S_{\bar{\eta}}$ versus the period $T_{n}$ of each $n$th wave component. The 


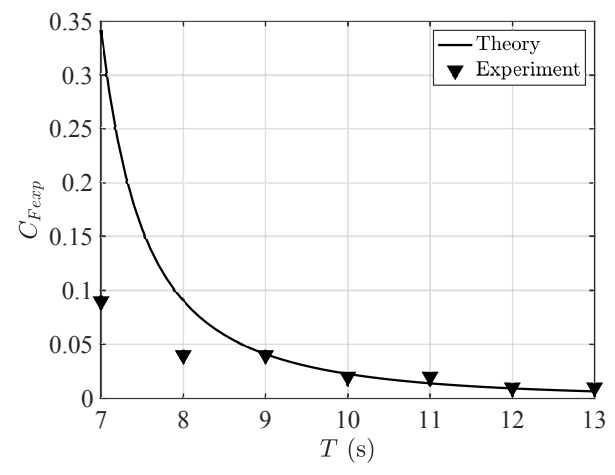

(a)

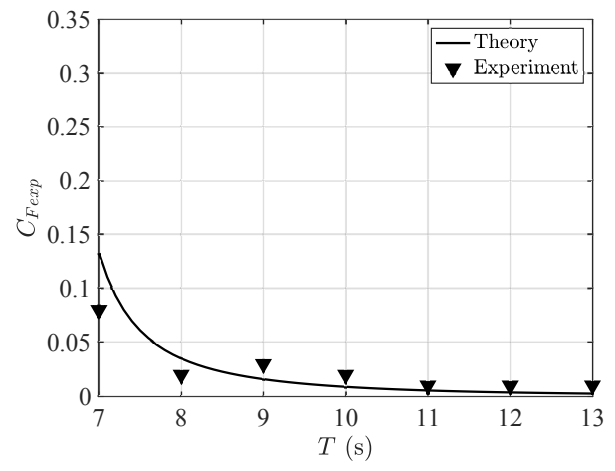

(b)

Figure 7: Behaviour of the capture factor $C_{F \exp }$ versus incident wave period $T$ in prototype values for two orifice diameters. 7 (a) $d_{0}=0.015 \mathrm{~m}, 7(\mathrm{~b}) d_{0}=0.019 \mathrm{~m}$. The solid line indicates the analytical results given by expression (98), while the triangular markers correspond to the experimental results of Perez-Collazo et al. (2018b).

theory predicts one peak around $T_{n}=5.5 \mathrm{~s}$, while the experimental response spectrum tends to decay towards small periods. As in the case of regular waves, this discrepancy is due to the linearised theory that tends to overestimate the amplitude response in resonance conditions. Indeed, the peak is located in correspondence of the Helmholtz pumping mode eigenfrequency. Beyond $T_{n}=5.5 \mathrm{~s}$ we stay in the range of validity of the scales (92) and good matching between theory and experiment is obtained.

\section{Conclusions}

We developed a linearised theory for a cylindrical OWC installed in hybrid wind-wave energy systems. The novel OWC model presented here has a skirt structure integral with the OWC whose task is to increase power extraction efficiency.

We evaluated the dependence of the hydrodynamic quantities such as added inertia, radiation damping and exciting force on the incident wave frequency. Our results show that large resonant peaks occur in correspondence of the frequencies very close to the eigenfrequencies of a cylindrical tank having depth equal to $h$. Furthermore, we performed a numerical check of the latter quantities and therefore of the accuracy of the results by deriving some useful integral identities based on Green's theorem. 


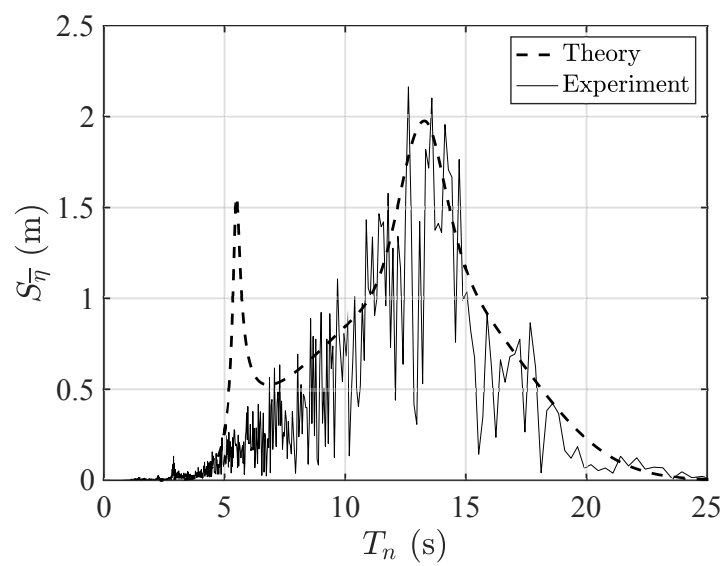

Figure 8: Behaviour of the response amplitude spectrum $S_{\bar{\eta}}$ versus the $n$th wave component period $T_{n}$ in prototype values for $H_{s}=3.5 \mathrm{~m}$ and peak period $T_{p}=13.3 \mathrm{~s}$. The dashed line represents the analytical results given by (99), while the continuous line corresponds to the spectrum of the time series obtained by Perez-Collazo et al. (2018b).

Then we investigated the effects of the skirt height and opening angle on the hydrodynamic behaviour and efficiency. We found that the greater the skirt height, the greater the efficiency when the Helmholtz pumping resonates while the narrow sloshing resonant peaks are almost unaffected and maintain their shape. This means that the sloshing dynamics depend mainly on the internal and external OWC radius. Indeed, we showed that when an internal cylinder is present, wide peaks on the capture factor behaviour can be obtained at large frequencies. Concerning the skirt opening angle, we obtained that the optimal configuration maximizing power extraction corresponds to the symmetric case $\theta_{1}=2 \pi / 3 ; \theta_{2}=\pi / 2 \mathrm{rad}$.

We also investigated the OWC response to random incident waves described by the JONSWAP spectrum. We showed that the presence of a broad range of wave frequencies does not couple well with the narrow resonant peaks of some sloshing modes. This is less true for the broad band Helmholtz-mode at low frequencies. In this case we have large radiation damping and the resonant peak almost keeps its shape. Outside resonance the efficiency is larger or comparable to that for the monochromatic case and the benefits of random waves are evident. Similar results are already well known for flap-type OWSCs in open sea.

Subsequently, we validated the analytical model with the experimental 
set-up developed by Perez-Collazo et al. (2018b). First, we derived the nonlinear boundary condition on the free surface inside the air chamber. This condition is completely generalised and therefore valid for any OWC laboratory model that uses orifices to simulate the presence of a turbine. We solved the problem by applying the perturbation expansion to the velocity potential and showed that the air pressure and the corresponding airflow through the orifice depend mainly on the diffraction potential at the leading order. We evaluated the corresponding theoretical capture factor and compared it with that obtained experimentally by Perez-Collazo et al. (2018b). Good agreement between both models was found especially for large incident wave periods and large orifice diameters. Finally, we compared theory and experiments by analysing the response spectra of the free-surface amplitude inside the OWC chamber in irregular waves. Good matching was obtained for frequencies not close to the resonant Helmholtz pumping mode.

\section{Funding source}

This work was supported by a Royal Society - CNR International Fellowship (Grant NF170771).

\section{References}

\section{References}

Astariz, S., Vazquez, A., Iglesias, G., 2015. Evaluation and comparison of the levelized cost of tidal, wave, and offshore wind energy. J. Renew. Sustain. Ener. 7, 1-11. (doi:10.1063/1.4932154)

Astariz, S., Vazquez, A., Iglesias, G., 2016. Wave energy vs. other energy sources: a reassessment of the economics. Int. J. Green Energy 13, 744755. (doi:10.1080/15435075.2014.963587)

Babarit, A., Hals, J., Muliawan, M., Kurniawan, A., Moan, T., Krokstad, J., 2012. Numerical benchmarking study of a selection of wave energy converters. Renew. Energy 41, 44-63. (doi:10.1016/j.renene.2011.10.002)

Babarit A., 2018. Ocean wave energy conversion. Elsevier. 
Deng, Z., Huang, Z., Law, A.W.K., 2013. Wave power extraction by an axisymmetric oscillating-water-column converter supported by a coaxial tube-sector-shaped structure. Appl. Ocean. Res. 42, 114-123. (doi:10.1016/j.apor.2013.05.006)

Deng, Z., Huang, Z., Law, A.W.K., 2014. Wave power extraction from a bottom-mounted oscillating water column converter with a v-shaped channel. Proc. R. Soc. Lond. A 470, 20140074. (doi:10.1098/rspa.2014.0074)

Evans, D.V., 1982. Wave power absorption by systems of oscillating surface pressure distributions. J. Fluid Mech. 114, 481-499. (doi:10.1017/S0022112082000263)

Evans, D.V., Porter, R., 1997. Efficient calculation of hydrodynamic properties of owc-type devices. J. Offshore Mech. Arctic Engng. 119, 210-218.

Falnes, J., 2002. Ocean waves and oscillating systems. Cambridge University Press.

Garrett, J.C.R., 1982. Bottomless harbours. J. Fluid Mech. 43, 433-449. (doi:10.1017/S0022112070002495)

Goda, Y., 2000. Random Seas and Design of Maritime Structures. World Scientific, Singapore.

Linton, C.M., McIver, P., 2001. Mathematical techniques for wave/structure interactions. Chapman \& Hall/CRC .

López, I., Pereiras, B., Castro, F., Iglesias, G., 2016. Holistic performance analysis and turbine-induced damping for an OWC wave energy converter. Renew. Energ. 85, 1155-1163. (doi:10.1016/j.renene.2015.07.075)

López, I., Pereiras, B., Castro, F., Iglesias, G., 2014. Optimisation of turbine-induced damping for an OWC wave energy converter using a RANS-VOF numerical model. Appl. Energ. 127, 105-114. (doi:10.1016/j.apenergy.2014.04.020)

Lovas, S., Mei, C.C., Liu, Y., 2010. Oscillating water column at a coastal corner for wave power extraction. Appl. Ocean. Res. 32, 267-283. (doi:10.1016/j.apor.2010.06.004) 
Magagna, D., Uihlein, A., 2015. Ocean energy development in Europe: Current status and future perspectives. Int. J. Mar. Energy. 11, 84-104. (doi:10.1016/j.ijome.2015.05.001)

Martin-Rivas, H., Mei, C.C., 2009a. Wave power extraction from an oscillating water column along a straight coast. Ocean Eng. 36, 426-433. (doi:10.1016/j.oceaneng.2009.01.009)

Martin-Rivas, H., Mei, C.C., 2009b. Wave power extraction from an oscillating water column at the tip of a breakwater. J. Fluid Mech. 629, 394-414. (doi:10.1017/S0022112009005990)

Mavrakos, S.A., 1985. Wave loads on stationary floating bottomless cylindrical body with finite wall thickness. Appl. Ocean. Res. 7, 213-224. (doi:10.1016/0141-1187(85)90028-8)

McCormick, M.E., 1981. Ocean wave energy conversion. Wiley Interscience.

Mei, C.C., Stiassnie, M., , D. K.-P., 2005. Theory and applications of ocean surface waves. World Scientific, Singapore.

Michele, S., Sammarco, P., d'Errico, M., Renzi, E., Abdolali, A., Bellotti, G., Dias, F., 2015. Flap gate farm: from Venice lagoon defense to resonating wave energy production. Part2: Synchronous response to incident waves in open sea. Appl. Ocean Res. 52, 43-61. (doi:10.1016/j.apor.2015.05.002)

Michele, S., Sammarco, P., d'Errico, M., 2016a. The optimal design of a flap gate array in front of a straight vertical wall: Resonance of the natural modes and enhancement of the exciting torque. Ocean Eng. 118, 152-164. (doi:10.1016/j.oceaneng.2016.04.002)

Michele, S., Sammarco, P., d'Errico, M., 2016b. Theory of the synchronous motion of an array of floating flap gates oscillating wave surge converter. Proc. R. Soc. Lond. A 472, 20160174. (doi:10.1098/rspa.2016.0174)

Michele, S., Sammarco, P., d'Errico, M., 2018. Weakly nonlinear theory for oscillating wave surge converters in a channel. J. Fluid Mech. 834, 55-91. (doi:10.1017/jfm.2017.724)

Michele, S., Renzi, E., Sammarco, P., 2019. Weakly nonlinear theory for a gate-type curved array in waves. J. Fluid Mech. 869, 238-263. (doi:10.1017/jfm.2019.223) 
Michele, S., Renzi, E., 2019. A second-order theory for an array of curved wave energy converters in open sea J. Fluid Struct. 88, 315-330. (doi:10.1016/j.jfluidstructs.2019.05.007)

Pereiras, B., López, I., Castro, F., Iglesias, G., 2015. Non-dimensional analysis for matching an impulse turbine to an owc (oscillating water column) with an optimum energy transfer. Energy 87, 481-489. (doi:10.1016/j.energy.2015.05.018)

Perez-Collazo, C., Greaves, D., Iglesias, G., 2015. A review of combined wave and offshore wind energy. Renew. Sust. Eenerg. Rev. 42, 141-153. (doi:10.1016/j.rser.2014.09.032)

Perez-Collazo, C., Greaves, D., Iglesias, G., 2018a. Hydrodynamic response of the wec sub-system of a novel hybrid wind-wave energy converter. Energ. Convers. Manage. 171, 307-325. (doi:10.1016/j.enconman.2018.05.090)

Perez-Collazo, C., Greaves, D., Iglesias, G., 2018b. A novel hybrid windwave energy converter for jacket frame substructures. Energies 11, 637. (doi:10.3390/en11030637)

Sammarco, P., Michele, S., d'Errico, M., 2013. Flap gate farm: from Venice lagoon defense to resonating wave energy production. Part1: Natural modes. Appl. Ocean Res. 43, 206-213. (doi:10.1016/j.apor.2013.10.001)

Sammarco, P., Tran, H.H., Mei, C.C., 1997a. Subharmonic resonance of Venice gates in waves. Part 1. Evolution equation and uniform incident waves. J. Fluid Mech. 349, 295-325. (doi:10.1017/S0022112097006848)

Sammarco, P., Tran, H.H., Gottlieb, O., Mei, C.C., 1997b. Subharmonic resonance of Venice gates in waves. Part 2. Sinusoidally modulated incident waves. J. Fluid Mech. 349, 295-325. (doi: 10.1017/S0022112097006836)

Sarkar, D., Renzi, E., Dias, F., 2014. Wave farm modelling of oscillating wave surge converters. Proc. R. Soc. Lond. A 470, 20140118. (doi:10.1098/rspa.2014.0118)

Sarmento, A.J.N.A., Falcão, A. F. de O., 1985. Wave generation by an oscillating surface-pressure and its applications in wave-energy extraction. J. Fluid Mech. 150, 467-485. (doi:10.1017/S0022112085000234) 
Xu, C., Huang, Z., Deng, Z., 2016. Experimental and theoretical study of a cylindrical oscillating water column device with a quadratic power take-off model. Appl. Ocean. Res. 57, 19-29. (doi.org/10.1016/j.apor.2016.02.003)

Xu, C., Huang, Z., 2019. Three-dimensional CFD simulation of a circular OWC with a nonlinear power-takeoff: Model validation and a discussion on resonant sloshing inside the pneumatic chamber. Ocean Eng. 176, 184198. (doi.org/10.1016/j.oceaneng.2019.02.010)

Zheng, S., Zhang, Y., Iglesias, G., 2018. Wave-structure interaction in hybrid wave farms. J. Fluid Struct. 83, 386-412. (doi:10.1016/j.jfluidstructs.2018.09.012)

Zheng, S., Zhang, Y., Iglesias, G., 2019. Coast/breakwaterintegrated owc: A theoretical model. Mar. Struct.. 66, 121-135. (doi:10.1016/j.marstruc.2019.04.001)

Zhou, Y., Zhang, C., Ning, D., 2018. Hydrodynamic investigation of a concentric cylindrical owc wave energy converter. Energies 11, 95. (doi:10.3390/en11040985) 